\title{
Design and Antiproliferative and Antioxidant Activities of Furan-Based Thiosemicarbazides and 1,2,4-Triazoles: Their Structure-Activity Relationship and SwissADME Predictions
}

\section{Yusuf SICAK ( $\nabla$ yusufsicak@mu.edu.tr)}

Mugla Sitki Kocman University: Mugla Sitki Kocman Universitesi https://orcid.org/0000-0003-23395837

\section{Research Article}

Keywords: Thiosemicarbazide, 1,2,4-Triazole, HeLa cancer, Antioxidant activity, SwissADME

Posted Date: April 19th, 2021

DOI: https://doi.org/10.21203/rs.3.rs-392722/v1

License: (1) This work is licensed under a Creative Commons Attribution 4.0 International License. Read Full License 


\section{Abstract}

Due to the limited number of drugs in current clinical use, the diverse biological applications of furan have encouraged the preparation of a wide variety of thiosemicarbazide and triazole derivatives for the purpose of developing new drug agents. In this study aimed to investigate the antiproliferative and antioxidant activities of some thiosemicarbazides (1-12) and 1,2,4-triazoles (13-24). Compound 15 (IC $_{50}$ : $8.81 \pm 0.28 \mu \mathrm{M}$ ) showed the highest antiproliferative activity against the cervical (HeLa) cancer cell line among the compounds. Compounds 15, 20, 21, and 22 of the 1,2,4-triazole derivatives (13-24) exhibited excellent antioxidant activity. Moreover, the physicochemical properties, pharmacokinetic properties, drug similarity, and medicinal chemistry properties of all synthesized products were calculated using SwissADME. In addition, the effect of the structure-activity relationships of the 1,2,4-triazole derivatives (13-24) on the results of antiproliferative and antioxidant activity assays was evaluated.

\section{Introduction}

Cancer, which has been one of the world's biggest health problems for many years, is a malignant disease of the cell cycle that involves uncontrollable mitosis of abnormal cells, which invade surrounding tissues and often spread to other parts of the body [1,2]. About 11 million cases of cancer are diagnosed each year. Cancer, if not treated correctly, is likely to become widespread in a large proportion of the world's population [3, 4]. The cervical cancer cell line known as HeLa, which was taken from Henrietta Lack, who passed away in 1951, is the oldest and most common cell line affecting women worldwide and cervical cancer ranks fourth in terms of both incidence and mortality $[5,6]$. The main treatments of the disease are surgery and radiotherapy. Surgery is performed if the disease is caught in the early stage of the disease, while radiotherapy is used for advanced stages. In the many clinical studies on cervical cancer, satisfactory results have been obtained with chemotherapy such as increasing the five-year survival rate of patients $[7,8]$. Since the incidence of cervical cancer is at an undeniable level in young women, chemotherapeutic agents are needed [9]. However, despite superior studies on the design of effective chemotherapeutic drugs, there are still drawbacks involving toxicity and selectivity $[10,11]$. The problem of toxicity and resistance of cancer cells to anticancer agents has led to a continuous search for new chemotherapy agents. High amounts of reactive oxygen species (ROS) have been reported to promote many aspects of tumor development and progression in almost all types of cancer [12].

ROS produced by the cellular metabolism in living cells, while essential for life, can adversely cause the destruction of tissues or affect their normal functioning [13]. By attacking healthy cells, ROS can change cell structure or cause the cell to lose its ability to function [14]. All ROS form strand breaks or damaged bases because they have the potential to interact with DNA cellular components found in the genetic material to match its unstable free electron [13]. ROS-induced oxidative damage plays an important role in the development of diseases such as neurodegenerative disorders, arthritis, arteriosclerosis, inflammation, weakening of the immune system, liver disease, brain dysfunction, cardiovascular events, diabetes, and kidney failure [15]. Furthermore, many researchers suggest that ROS damage plays a vital role particularly in the development of malignant cancer and the initiation of proliferation of cancerous 
cells [13]. Antioxidant defense system agents developed for the elimination or cleaning of ROS in order to prevent permanent diseases caused by ROS can prevent oxidative chain reactions, direct quenching of reactive oxygen species, enzyme inhibition, reactions responsible for chelating metal ions such as $\mathrm{Fe}^{+2}$ and $\mathrm{Cu}^{+}$, and free radical-mediated oxidative damage of biomolecules such as proteins, nucleic acids, polyunsaturated lipids, and sugars even at low concentrations [16]. Therefore, there is a need to develop radical scavenging antioxidant agents to control the harmful effects of free radicals in the human body.

Heterocyclic compounds are compounds in which the ring structure contains atoms such as nitrogen, oxygen, and sulfur and they are capable of various interactions including hydrogen bond donor/acceptor capacity, pi stacking, ability to bind to enzymes, and forming coordination bonds with metals as well as Van der Waals and hydrophobic forces $[17,18]$. Many enzyme binding pockets undergo interactions with heterocyclic structures and heterocyclic compounds have versatile functionality and alternatives to numerous biological pathways. Thanks to the superior properties of heterocyclic compounds such as treating cancer or disrupting the biological pathways related to the progression of cancer, the design of synthetic molecules of heterocyclic-based chemotherapy agents has been kept up to date [18]. In particular, furan-derived rings are an important class of heterocyclic compounds with very important biological properties. Many researchers have shown intense interest in the synthesis of a new furanderived scaffold with different pharmacological activities for the discovery of new drugs in recent decades [19]. On the other hand, 1,2,4-triazole $\left(\mathrm{C}_{2} \mathrm{~N}_{3} \mathrm{H}_{3}\right)$ derivatives containing three nitrogen atoms from five-membered ring systems, which were firstly described by Blodin, have become the focus of attention of many research teams for obtaining synthetic agents with high bioavailability due to the known antidepressant, anticancer, anti-inflammatory, analgesic, antiviral, and antioxidant effectiveness of these compounds [20-22].

To date, all triazoles recorded in the literature have been of synthetic origin, and there is no study showing that a triazole and its derivative isolated from natural products have been detected [23]. The synthesis design strategy of the present study, inspired by the known anticancer therapeutic effect of 1,2,4-triazole, due to the need to discover alternative agent(s) for cancer, which is the biggest health problem of the age, started with the synthesis of some thiosemicarbazide derivatives (1-12) from heterocyclic-based 2furanoylhydrazide, followed by the synthesis of the activity potential agent 1,2,4-triazole derivatives (1324). After the structures of all the derivatives synthesized were elucidated, their in vitro antiproliferative activity against the HeLa cancer cell line and in vitro antioxidant activity were tested. In addition, the physicochemical properties (including Lipinski), pharmacokinetics, drug-likeness, and medicinal chemistry properties of products synthesized (1-24) were calculated using the program SwissADME.

\section{Results And Discussion}

\section{Chemistry}

This study is the first to determine the in vitro antiproliferative activity against HeLa cancer cells and antioxidant activity of thiosemicarbazides (1-12) and triazoles (13-24). The synthetic route to prepare the 
thiosemicarbazides (1-12) and triazoles (13-24), the target molecules, is given in Fig. 1.

In the IR spectra, $\mathrm{v}(\mathrm{N}-\mathrm{H})$ stretching bands of thiosemicarbazide derivates 1-12 for $\mathrm{CONH}, \mathrm{PhNH}$, and CSNH were observed at 3748-3629 $\mathrm{cm}^{-1}, 3351-3211 \mathrm{~cm}^{-1}$, and 3211-3117 $\mathrm{cm}^{-1}$, respectively [24]. In addition, characteristic $\mathrm{v}(\mathrm{C}=0)$ and $\mathrm{v}(\mathrm{C}=\mathrm{S})$ stretching bands were determined at $1689-1647 \mathrm{~cm}^{-1}$ and $1118-1012$ $\mathrm{cm}^{-1}$, respectively. Moreover, the stretching bands of the $\mathrm{N}-\mathrm{H}$ group was determined to be absorption at $2600-2550 \mathrm{~cm}^{-1}$. The $\mathrm{v}(\mathrm{N}-\mathrm{H})$ stretching band for CSNH of triazole derivates 13-24 was observed at 3276$3056 \mathrm{~cm}^{-1}$. The $\mathrm{v}(\mathrm{N}-\mathrm{H})$ stretching band of $\mathrm{CONH}$ and $\mathrm{PhNH}$ observed for thiosemicarbazide derivates 1-12 disappeared in triazoles 13-24.

In the ${ }^{1} \mathrm{H}$ NMR spectra of the thiosemicarbazides 1-12 and 1,2,4-triazole compounds 13-24, the protons of the phenyl ring and its substituents in compounds 1-24 appeared to be resonance in the expected regions. Moreover, the protons $(\mathrm{H}-1, \mathrm{H}-2$, and $\mathrm{H}-3)$ of the furan ring in compounds $1-12$ were found to have resonance at 7.78-8.02, 6.31-6.70, and 7.23-7.29 ppm, respectively, while the protons $(\mathrm{H}-1, \mathrm{H}-2$, and $\mathrm{H}-3)$ of the furan ring in compounds 13-24 were found to have resonance at 7.73-8.14, 6.31-6.70, and 5.86-6.30 ppm, respectively. In general, the aromatic protons $(\mathrm{H}-11, \mathrm{H}-12$, and $\mathrm{H}-13)$ were observed between $\delta 6.60$ and $7.87 \mathrm{ppm}$ for thiosemicarbazides 1-12. The $\mathrm{NH}(\mathrm{H}-6, \mathrm{H}-7$, and $\mathrm{H}-9)$ protons of thiosemicarbazide (-NH$\mathrm{NH}-\mathrm{C}=\mathrm{S}-\mathrm{NH}-$ ) derivatives 1-12 were detected at $\delta$ 9.11-10.03, 10.34-11.29, and 9.55-10.52 ppm, respectively. In the a study [25], ${ }^{1} \mathrm{H}$ NMR protons data in the thiosemicarbazide scaffold were found to be compatible with the chemical shift values of thiosemicarbazide protons in this study. The resonance of proton $\mathrm{H}-7$ in the 1,2,4-triazole derivatives at 13.97-14.22 ppm and the disappearance of the $\mathrm{H}-6$ and $\mathrm{H}-9$ protons in the thiosemicarbazide derivatives in the cyclization products were definite evidence of the synthesis of 1,2,4-triazole derivatives. It has been reported in the literature that the $\mathrm{NH}$ proton of the 1,2,4triazole ring resonates at 13.99-14.01 ppm [26].

In the ${ }^{13} \mathrm{C}$ NMR spectra of thiosemicarbazides $1-12$, carbon resonances of the $\mathrm{C}=\mathrm{O}$ and $\mathrm{C}=\mathrm{S}$ groups were determined at $158.02-159.13 \mathrm{~cm}^{-1}$ and $180.63-182.27 \mathrm{~cm}^{-1}$, respectively $\mathrm{In}$ the study conducted by [27], it was determined that the ${ }^{13} \mathrm{C}$ NMR spectra of $\mathrm{C}=\mathrm{O}$ and $\mathrm{C}=\mathrm{S}$ chemical shift values in the thiosemicarbazide scaffold were in similar ranges with the $\mathrm{C}=\mathrm{O}$ and $\mathrm{C}=\mathrm{S}$ chemical shift values of thiosemicarbazide derivatives (1-12) in this study. In the ${ }^{13} \mathrm{C}$ NMR spectra, the $\mathrm{C}-5$ carbon of azomethine $(-\mathrm{C}=\mathrm{N}-)$ and the $\mathrm{C}_{8}$ carbon of thioxo (-C=S-) of 1,2,4-triazole derivatives $13-24$ resonated at $\delta 145.69-162.28$ ppm and $168.82-$ $169.57 \mathrm{ppm}$. The aromatic carbons were observed between $\delta 102.07$ and $156.66 \mathrm{ppm}$ for 1,2,4-triazole derivatives 13-24, while C-1, C-2, C-3, and C-4 of the furan ring of 1,2,4-triazole derivatives 13-24 were observed at $\delta 140.17-144.25 \mathrm{ppm}, 111.51-112.43 \mathrm{ppm}, 111.82-113.31 \mathrm{ppm}$, and 143.37-145.97 ppm, respectively. The aromatic protons and carbons were shifted up or down by the influence of electronwithdrawing or electron-donating groups connected to the aromatic ring.

Three spin systems were observed in the COSY spectrum $(\mathrm{H}-14-\mathrm{H}-15, \mathrm{H}-11-\mathrm{H}-12)$ of compound 24 , which was chosen as a model compound for the 2D NMR spectrum (Fig. 2). 
The HMQC spectrum of compound 24 showed that $\mathrm{H}-1$ was in correlation with $\mathrm{C}-1$, and $\mathrm{H}-2$ with $\mathrm{C}-2, \mathrm{H}-3$ with $\mathrm{C}-3, \mathrm{H}-5$ with $\mathrm{C}-8, \mathrm{H}-6$ with $\mathrm{C}-9, \mathrm{H}-7$ with $\mathrm{C}-11$, and $\mathrm{H}-8$ with $\mathrm{C}-12$ (Fig. 3).

The values obtained from the elemental analysis of thiosemicarbazides 1-12 and 1,2,4-triazole derivatives 13-24 were compatible with the calculated values.

\section{Pharmacology}

\section{Antiproliferative activity of the synthesized compounds}

The antiproliferative activities of compounds 1-24 against HeLa cell lines were investigated at four concentrations $(100,50,25$, and $5 \mu \mathrm{M})$. The $\mathrm{IC}_{50}$ values of compounds 1-24 against HeLa are given at Table 1. The anticancer activity of 1-24 increased with the increasing concentration against the HeLa cell lines. The antiproliferative activity of 1,2,4-triazole derivatives 13-24 against the HeLa cells was almost twice as high as that of thiosemicarbazide derivatives 1-12 obtained by ring closure of thiosemicarbazide derivatives. Compound $\mathbf{3}\left(\mathrm{IC}_{50}: 19.83 \pm 0.42 \mu \mathrm{M}\right)$ from thiosemicarbazide derivatives 1-12 showed the highest antiproliferative activity against HeLa cancer cells. Among the 1,2,4-triazole derivatives 13-24 compound $15\left(\mathrm{IC}_{50}: 8.81 \pm 0.28 \mu \mathrm{M}\right)$ exhibited the highest activity. Additionally, compound 18 displayed moderate activity with an $\mathrm{IC}_{50}$ value of $27.31 \pm 0.05 \mu \mathrm{M}$, followed by compound $16\left(\mathrm{IC}_{50}\right.$ : $\left.30.54 \pm 0.19 \mu \mathrm{M}\right)$.

\section{Antioxidant activity of the synthesized compounds}

The in vitro antioxidant activities of thiosemicarbazides (1-12) and 1,2,4-triazoles (13-24) were determined by four complimentary assays, namely $\beta$-carotene-linoleic acid, ABTS ${ }^{+}$scavenging, CUPRAC, and DPPH scavenging methods. Generally, the antioxidant activity of 1,2,4-triazoles (13-24) was higher than that of the thiosemicarbazides derivatives (1-12). The antioxidant activity results of compounds 124 are shown in Table 2. According to these assay results, compounds $3, \mathbf{1 0}$, and $\mathbf{9}$ of the thiosemicarbazide derivatives (1-12) demonstrated the highest lipid peroxidation inhibitory activity $\mathrm{IC}_{50}$ values of $21.80 \pm 0.69,26.49 \pm 0.61$, and $29.07 \pm 0.52 \mu \mathrm{M}$, respectively, while compounds $15,18,19,20,21$, and 22 of the 1,2,4-triazole derivatives (13-24) exhibited the highest lipid peroxidation inhibitory activity. Among all the synthetic products (1-24), compounds $\mathbf{3}, \mathbf{9}$, and $\mathbf{1 0}$ of the thiosemicarbazide series and compounds $15\left(\mathrm{IC}_{50}: 9.38 \pm 0.93 \mu \mathrm{M}\right), 22\left(\mathrm{IC}_{50}: 14.79 \pm 0.26 \mu \mathrm{M}\right), 21\left(\mathrm{IC}_{50}: 19.88 \pm 0.75 \mu \mathrm{M}\right), 20\left(\mathrm{IC}_{50}\right.$ : $21.35 \pm 0.14 \mu \mathrm{M}), \mathbf{1 8}\left(\mathrm{IC}_{50}: 23.96 \pm 0.24 \mu \mathrm{M}\right)$, and $19\left(\mathrm{IC}_{50}: 26.74 \pm 0.66 \mu \mathrm{M}\right)$ of the 1,2,4-triazole series showed the best cation radical scavenging activity against ABTS.+ ${ }^{+}$. Compound 3 of the thiosemicarbazide series (1-12) and compound 15 of the 1,2,4-triazole derivatives (13-24) showed excellent antioxidant activity in all four assays. In addition, while the CUPRAC activity of all synthesized compounds was higher than that of a-TOC used as pharmaceutical standard in the assay, DPPH scavenging activity showed it was higher than that of BHT used as positive standard.

\section{SwissADME Prediction of synthesized compounds}


The data predicted for the physicochemical characteristics, lipophilicity, solubility, pharmacokinetics, drug likeness, and medicinal chemistry of synthesized compounds evaluated by SwissADME are given in Table S1 (see: supplementary file). According to Lipinski's rule of five, the molecular weights of thiosemicarbazide and 1,2,4-triazole derivatives were 261.30-363.74 and 243.28-345.73 Da, respectively, and within the limits of 200-600 Da. The logP values of the thiosemicarbazide (1-12) and 1,2,4-triazole (13-24) derivatives were less than 5 , in the range of 2.24-3.11. The HBA number of all synthesis products was between 2 and 5 and less than 10; the number of HBD atoms for the thiosemicarbazide (1-12) and 1,2,4-triazole (13-24) derivatives was 3 and 1, respectively, less than 5 [28].

Brain Or IntestinaL EstimateD permeation (BOILED-Egg) method is a graphical model that works by calculating the polarity and lipophilicity of small molecules. This prediction provides a visual clue to the synthesis design of new compounds in terms of the oral absorption potential of drug candidates [29]. Graphical estimations of gastrointestinal absorption and blood-brain barrier (BBB) penetration of the synthesized thiosemicarbazide and 1,2,4-triazole derivatives are shown in Fig. 4. According to the BOILED-Egg plot, none of the compounds synthesized were located in the BBB (except compound 13 of the 1,2,4-triazole derivatives) with the yellow circle expressing good intestinal absorption and the gray region representing poor intestinal absorption. All compounds (except compound 13) are contained within the while ellipse representing human intestinal absorption. Compound $\mathbf{1 0}$ was found to be the blue spot, evidence of its good bioavailability. Thus, compound $\mathbf{1 0}$ demonstrated that it could be a substrate for P-glycoprotein and it could reduce its absorption and penetration in the brain. All compounds except compound 13 can be promising agents that can very easily be absorbed by the gastrointestinal tract without potential BBB permeability. Since these compounds cannot cross the BBB, they do not cause central nervous system depression or drowsiness as side effects.

\section{Conclusions}

The current research focused mainly on the synthesis of some 1,2,4-triazole derivatives (13-24) and determination of the antiproliferative and antioxidant activities of the synthesized compounds. Based on the finding that the compounds can increase the absorption power of free radicals with their high nitrogen content, 1,2,4-triazole derivatives (13-24) were synthesized in order to prevent various disorders caused by free radicals. The synthetic route to the 1,2,4-triazoles (13-24) was based on cyclization of thiosemicarbazides (1-12). Of the series synthesized, compound 15 exhibited the best antiproliferative activity against cervical cell lines. According to the results of antioxidant capacity, compounds 15, 20, 21, and 22, which protect against lipid peroxidation and radical damage, may be drug candidates as antioxidant agents. It is observed that chemical structural variation in the synthesized molecules leads to different bioactivity and of course structural modification of the molecules changes the biological activity in a regular trend [30]. This situation, especially the substitution of chlorine or methoxy groups in the aromatic ring system, has been found to vary in anticancer and antioxidant activity. Based on these results, new studies in the field of medicinal chemistry on the modification of structures bearing $1,2,4$ triazole core can be pioneered. Consequently, the presence of 2,4-dichloro and 2,4 or 3,4-dimethoxy 
substituents in the phenyl ring attached to position 4 in the 1,2,4-triazole nucleus was found to increase antioxidant and anticancer activity.

\section{Materials And Methods}

\section{General}

Analytical grade chemicals and solvents were purchased from Acros, Alfa Aesar, Sigma-Aldrich, and Merck. Thin layer chromatography (TLC, Merck $60 \mathrm{~F}_{254}$ ) was used to monitor the chemical reactions. The melting points were examined using an SMP20 melting point apparatus and were uncorrected. The FTIR spectra were acquired using a Frontier spectrometer by attenuated total reflectance (ATR) apparatus (PerkinElmer, Waltham, Massachusetts, USA). Elemental analyses (CHNS) were performed on a Thermo Scientific Flash 2000 elemental analyzer (Finnigan MAT, USA). The ${ }^{1} \mathrm{H}-\mathrm{NMR},{ }^{13} \mathrm{C}-\mathrm{NMR}, \mathrm{COSY}$, and HMQC spectra were recorded on an Agilent Technologies $400 \mathrm{MHz}$ NMR spectrometer (Agilent, USA). The cell proliferation BrdU ELISA kits were supplied by Roche (Roche Diagnostic $\mathrm{GmbH}$, Germany). The FTIR and NMR data of compounds 1-24 are presented in the supplementary data section.

\section{Synthesis}

\section{General synthetic procedure for compounds 1-12}

2-Furoic acid hydrazide $(0.01 \mathrm{~mol})$ and substituted phenylisothiocyanate $(0.01 \mathrm{~mol})$ were dissolved in methanol and the mixture was refluxed overnight. After completion of the reaction, the mixture was left to cool down in order to precipitate the solids. Finally, ethanol was used to purify the precipitate [31].

\section{1-[(furan-2-carbonyl)]-4-[phenyl]thiosemicarbazide (1) [32]}

White solid. Yield: 73\%; m.p. 205-206 ${ }^{\circ} \mathrm{C}$. FTIR vmax $\left(\mathrm{cm}^{-1}\right)$ : 3659, 3317, 3135 (NH stretching), 3057 (ArCH stretching), 1670 (C=0), 1294 (C-N), 1068 (C=S). ${ }^{1} \mathrm{H}$ NMR (400 MHz, DMSO- $\left.d_{6}\right): \delta 6.88$ (d, $1 \mathrm{H}, J_{1}=3.2 \mathrm{~Hz}$, $\left.J_{2}=1.6 \mathrm{~Hz}, \mathrm{H}-2\right), 7.16(\mathrm{t}, 1 \mathrm{H}, J=7.2 \mathrm{~Hz}, \mathrm{H}-13), 7.25(\mathrm{~d}, 1 \mathrm{H}, J=3.2 \mathrm{~Hz}, \mathrm{H}-3), 7.33(\mathrm{t}, 2 \mathrm{H}, J=7.6 \mathrm{~Hz}, \mathrm{H}-12), 7.45$ (d, 2H, J=7.2 Hz, H-11), 7.92 (brs, 1H, H-1), 9.67 (brs, 1H, H-6), 9.82 (brs, $1 \mathrm{H}, \mathrm{H}-9$ ), 10.42 (brs, $1 \mathrm{H}, \mathrm{H}-7$ ). ${ }^{13} \mathrm{C}$ NMR (150 MHz, DMSO-d $d_{6}$ ): $\delta 112.33$ (C-2), 115.26 (C-3), 125.49 (C-13), 126.38 (C-11), 128.35 (C-12), 139.65 (C-10), 146.09 (C-1), 146.87 (C-4), 158.07 (C-5), 181.53 (C-8). Anal. calc. for $\left(\mathrm{C}_{12} \mathrm{H}_{11} \mathrm{~N}_{3} \mathrm{O}_{2} \mathrm{~S}\right)$ : C, $55.16 ; \mathrm{H}, 4.24 ; \mathrm{N}, 16.08 ; \mathrm{S}: 12.27 \%$; found: $\mathrm{C}, 55.10 ; \mathrm{H}, 4.16 ; \mathrm{N}, 16.10 ; \mathrm{S}: 11.98 \%$.

\section{1-[(furan-2-carbonyl)]-4-[4-chlorophenyl]thiosemicarbazide (2) [32]}

White solid. Yield: 71\%; m.p. 218-220 ${ }^{\circ} \mathrm{C}$. FTIR vmax $\left(\mathrm{cm}^{-1}\right)$ : 3671, 3293, 3180 (NH stretching), 2989 (ArCH stretching), 1668 (C=0), 1300 (C-N), 1090 (C=S), 1013 (C-Cl). ${ }^{1} \mathrm{H}-\mathrm{NMR}\left(400 \mathrm{MHz}, \mathrm{DMSO}-d_{6}\right): \delta 6.68$ (dd, $\left.1 \mathrm{H}, J_{1}=3.6 \mathrm{~Hz}, J_{2}=1.6 \mathrm{~Hz}, \mathrm{H}-2\right), 7.25(\mathrm{~d}, 1 \mathrm{H}, J=3.2 \mathrm{~Hz}, \mathrm{H}-3), 7.38$ (d, 2H, J=8.8 Hz, H-11), 7.49 (d, 2H, J=8.0 $\mathrm{Hz}, \mathrm{H}-12), 7.92$ (d, 1H, J=0.8 Hz, H-1), 9.77 (s, 1H, H-6), 9.86 (brs, 1H, H-9), 10.44 (s, 1H, H-7). ${ }^{13} \mathrm{C}$ NMR (150 MHz, DMSO- $d_{6}$ ): $\delta 112.36$ (C-2), 115.35 (C-3), 127.93 (C-11), 128.26 (C-13), 129.51 (C-12), 138.66 (C-10), 
146.15 (C-1), 146.81 (C-4), 158.09 (C-5), 181.53 (C-8). Anal. calc. for $\left(\mathrm{C}_{12} \mathrm{H}_{10} \mathrm{ClN}_{3} \mathrm{O} 2 \mathrm{~S}\right)$ : C, 48.73; H.3.41; N, 14.21; S: $10.84 \%$; found: C, 47.99; H, 3.40; N, 14.17; S: 10.75\%.

\section{1-[(furan-2-carbonyl)]-4-[3,5-(dichloro)phenyl]thiosemicarbazide (3)}

White solid. Yield: 90\%; m.p. 211-213 ${ }^{\circ} \mathrm{C}$. FTIR vmax $\left(\mathrm{cm}^{-1}\right)$ : 3667, 3316, 3209 (NH stretching), 2988 (ArCH stretching), 1672 (C=0), 1298 (C-N), 1118 (C=S), 1015 (C-Cl). ${ }^{1} \mathrm{H}-\mathrm{NMR}$ (400 MHz, DMSO-d $)_{6}$ ): $\delta 6.68$ (dd, $\left.1 \mathrm{H}, J_{1}=3.2 \mathrm{~Hz}, J_{2}=1.6 \mathrm{~Hz}, \mathrm{H}-2\right), 7.26(\mathrm{~d}, 1 \mathrm{H}, \mathrm{J}=3.2 \mathrm{~Hz}, \mathrm{H}-3), 7.37$ (brs, $\left.1 \mathrm{H}, \mathrm{H}-13\right), 7.71$ (s, 2H, H-11), 7.94 (brs, $1 \mathrm{H}, \mathrm{H}-1), 9.94$ (brs, $1 \mathrm{H}, \mathrm{H}-6), 10.02$ (brs, $1 \mathrm{H}, \mathrm{H}-9), 10.49$ (brs, $1 \mathrm{H}, \mathrm{H}-7) .{ }^{13} \mathrm{C}$ NMR $\left(150 \mathrm{MHz}, \mathrm{DMSO}-d_{6}\right)$ : $\delta 112.44$ (C-2), 115.57 (C-3), 123.69 (C-13), 124.48 (C-11), 133.40 (C-12), 142.13 (C-10), 146.27 (C-1), 146.69 (C-4), 158.11 (C-5), 181.20 (C-8). Anal. calc. for $\left(\mathrm{C}_{12} \mathrm{H}_{9} \mathrm{Cl}_{2} \mathrm{~N}_{3} \mathrm{O}_{2} \mathrm{~S}\right)$ : C, 43.65; $\mathrm{H}, 2.75 ; \mathrm{N}, 12.73$; S: 9.71\%; found: C, $44.01 ; \mathrm{H}, 2.70 ; \mathrm{N}, 12.76$; $\mathrm{S}: 9.88 \%$.

\section{1-[(furan-2-carbonyl)]-4-[\{4-chloro-3-(trifluoromethyl)phenyl\}]thiosemicarbazide (4)}

White solid. Yield: 84\%; m.p. 222-224 ${ }^{\circ} \mathrm{C}$. FTIR vmax $\left(\mathrm{cm}^{-1}\right): 3671,3315,3211$ (NH stretching), 3084 (ArCH stretching), 1672 (C=0), 1321 (C-N), 1111 (C-F), 1080 (C=S), 1015 (C-Cl). ${ }^{1} \mathrm{H}-\mathrm{NMR}$ (400 MHz, DMSO- $d_{6}$ ): $\delta$ $6.70(\mathrm{~d}, 1 \mathrm{H}, J=1.6 \mathrm{~Hz}, \mathrm{H}-2), 7.27$ (d, 1H, J=2.8 Hz, H-3), 7.68 (d, 1H, J=8.4 Hz, H-14), 7.87-7.96 (m, 2H, H-11 and $\mathrm{H}-15), 8.02(\mathrm{~d}, 1 \mathrm{H}, \mathrm{J}=0.8 \mathrm{~Hz}, \mathrm{H}-1), 10.03(\mathrm{~s}, 1 \mathrm{H}, \mathrm{H}-6), 10.52(\mathrm{~s}, 1 \mathrm{H}, \mathrm{H}-9), 11.29(\mathrm{~s}, 1 \mathrm{H}, \mathrm{H}-7) .{ }^{13} \mathrm{C}$ NMR (150 MHz, DMSO- $d_{6}$ ): $\delta 112.45$ (C-2), 112.89 (C-11), 113.33 (C-3), 122.42 (C-15), 130.79 (C-13), 131.59 (C16), 132.96 (C-12), 139.08 (C-14), 146.28 (C-10), 146.72 (C-1), 151.82 (C-4), 159.13 (C-5), 181.41 (C-8). Anal. calc. for $\left(\mathrm{C}_{13} \mathrm{H}_{9} \mathrm{ClF}_{3} \mathrm{~N}_{3} \mathrm{O}_{2} \mathrm{~S}\right)$ : C, 42.93; $\mathrm{H}, 2.49 ; \mathrm{N}, 11.55 ; \mathrm{S}: 8.82 \%$; found: $\mathrm{C}, 42.86 ; \mathrm{H}, 2.39 ; \mathrm{N}, 11.62$; S: $8.75 \%$.

\section{1-[(furan-2-carbonyl)]-4-[\{5-chloro-2-(methoxy) phenyl\}]thiosemicarbazide (5) [32]}

White solid. Yield: 59\%; m.p. 209-211 ${ }^{\circ} \mathrm{C}$. FTIR vmax $\left(\mathrm{cm}^{-1}\right)$ : 3672, 3264, 3143 (NH stretching), 2989 (ArCH stretching), 1668 (C=0), 1315 (C-N), 1086 (C=S), 1020 (C-Cl). ${ }^{1} \mathrm{H}-\mathrm{NMR}\left(400 \mathrm{MHz}, \mathrm{DMSO}-d_{6}\right.$ ): $\delta 3.76$ (s, 3H, $\mathrm{H}-16), 6.69$ (d, 1H, J=1.3 Hz, H-2), 7.08 (d, 1H, J=7.8 Hz, H-12), 7.21 (d, 1H, J=7.8 Hz, H-13), 7.29 (d, $1 \mathrm{H}$, J=3.3 Hz, H-3), 7.94 (brs, 2H, H-1 and H-15), 9.26 (brs, 1H, H-6), 9.93 (brs, $1 \mathrm{H}, \mathrm{H}-9$ ), 10.57 (brs, $1 \mathrm{H}, \mathrm{H}-7) .{ }^{13} \mathrm{C}$ NMR (150 MHz, DMSO- $d_{6}$ ): $\delta 56.57$ (C-16), 112.38 (C-2), 113.45 (C-12), 115.62 (C-3), 123.16 (C-15), 123.50 (C-13), 126.27 (C-14), 129.47 (C-10), 146.44 (C-1), 149.90 (C-4), 151.88 (C-11), 158.09 (C-5), 182.27 (C-8). Anal. calc. for $\left(\mathrm{C}_{13} \mathrm{H}_{12} \mathrm{CIN}_{3} \mathrm{O}_{3} \mathrm{~S}\right)$ : $\mathrm{C}, 47.93 ; \mathrm{H}, 3.71 ; \mathrm{N}, 12.90 ; \mathrm{S}: 9.84 \%$; found: $\mathrm{C}, 47.83 ; \mathrm{H}$, $3.61 ; \mathrm{N}, 12.93 ; \mathrm{S}: 9.82 \%$.

\section{1-[(furan-2-carbonyl)]-4-[2,4-(dimethoxy)phenyl]thiosemicarbazide (6)}

Cream solid. Yield: 37\%; m.p. 199-201 ${ }^{\circ} \mathrm{C}$. FTIR vmax $\left(\mathrm{cm}^{-1}\right)$ : 3285, 3186 (NH stretching), 2988 (ArCH stretching), 2836 (R-CH), 1647 (C=0), 1269 (C-N), 1027 (C=S). ${ }^{1} \mathrm{H}-\mathrm{NMR}$ (400 MHz, DMSO-d $d_{6}$ ): $\delta 3.74-3.83$ (m, 6H, H-16 and H-17), 6.51 (d, 1H, J=8.4 Hz, H-14), 6.60 (brs, 1H, H-2), 6.67 (brs, 1H, H-12), $7.26(\mathrm{~d}, 1 \mathrm{H}$, $J=2.8 \mathrm{~Hz}, \mathrm{H}-3$ ), 7.44 (d, 1H, J=8.4 Hz, H-15), 7.91 (brs, 1H, H-1), 9.11 (brs, 1H, H-6), 9.59 (brs, 1H, H-9), 
$10.43(\mathrm{~s}, 1 \mathrm{H}, \mathrm{H}-7) .{ }^{13} \mathrm{C}$ NMR $(150 \mathrm{MHz}$, DMSO-d 6 ): $\delta 55.74$ (C-16), 56.09 (C-17), 99.28 (C-12), 104.46 (C14), 112.27 (C-2), 115.32 (C-3), 121.42 (C-15), 129.16 (C-10), 146.19 (C-1), 146.69 (C-4), 151.42 (C-11), 157.08 (C-13), 158.12 (C-5), 182.00 (C-8). Anal. calc. for $\left(\mathrm{C}_{14} \mathrm{H}_{15} \mathrm{~N}_{3} \mathrm{O}_{4} \mathrm{~S}\right)$ : C, 52.33; $\mathrm{H}, 4.70 ; \mathrm{N}, 13.08 ; \mathrm{S}$ : 9.98\%; found: $C, 52.28 ; H, 4.69 ; N, 13.13 ;$ S: $9.95 \%$.

\section{1-[(furan-2-carbonyl)]-4-[2,5-(dimethoxy)phenyl]thiosemicarbazide (7)}

White solid. Yield: 77\%; m.p. 204-206 ${ }^{\circ} \mathrm{C}$. FTIR vmax $\left(\mathrm{cm}^{-1}\right)$ : 3658, 3211, 3117 (NH stretching), 2997 (ArCH stretching), 2832 (R-CH), 1668 (C=0), $1280(\mathrm{C}-\mathrm{N}), 1012$ (C=S). ${ }^{1} \mathrm{H}-\mathrm{NMR}\left(400 \mathrm{MHz}, \mathrm{DMSO}-d_{6}\right): \delta 3.70$ (s, $6 \mathrm{H}, \mathrm{H}-16$ and H-17), $6.70(\mathrm{~d}, 1 \mathrm{H}, \mathrm{J}=2.4 \mathrm{~Hz}, \mathrm{H}-2), 6.71(\mathrm{~d}, 1 \mathrm{H}, \mathrm{J}=9.2 \mathrm{~Hz}, \mathrm{H}-13), 6.96(\mathrm{~d}, 1 \mathrm{H}, \mathrm{J}=9.2 \mathrm{~Hz}, \mathrm{H}-12)$, $7.29(\mathrm{~d}, 1 \mathrm{H}, \mathrm{J}=3.2 \mathrm{~Hz}, \mathrm{H}-3), 7.78-7.94(\mathrm{~m}, 2 \mathrm{H}, \mathrm{H}-1$ and H-15), 9.17 (brs, $1 \mathrm{H}, \mathrm{H}-6), 9.86$ (brs, 1H, H-9), 10.55 (brs, $1 \mathrm{H}, \mathrm{H}-7) .{ }^{13} \mathrm{C}$ NMR $\left(150 \mathrm{MHz}, \mathrm{DMSO}-d_{6}\right): \delta 55.81(\mathrm{C}-17), 56.75$ (C-16), 110.45 (C-13), 111.94 (C-15), 112.38 (C-2), 112.69 (C-12), 115.59 (C-3), 128.90 (C-10), 146.38 (C-1), 146.48 (C-4), 148.45 (C-11), 152.87 (C-14), 158.09 (C-5), 180.63 (C-8). Anal. calc. for $\left(\mathrm{C}_{14} \mathrm{H}_{15} \mathrm{~N}_{3} \mathrm{O}_{4} \mathrm{~S}\right)$ : C, 52.33; $\mathrm{H}, 4.70 ; \mathrm{N}, 13.08 ; \mathrm{S}: 9.98 \%$; found: $C, 52.30 ; H, 4.72 ; N, 13.11 ; S: 9.93 \%$.

\section{1-[(furan-2-carbonyl)]-4-[3,4-(dimethoxy)phenyl]thiosemicarbazide (8)}

White solid. Yield: $66 \%$; m.p. 200-202 ${ }^{\circ} \mathrm{C}$. FTIR vmax $\left(\mathrm{cm}^{-1}\right)$ : 3629, 3310, 3125 (NH stretching), 2957 (ArCH stretching), 2836 (R-CH), 1686 (C=0), 1280 (C-N), 1023 (C=S). ${ }^{1} \mathrm{H}-\mathrm{NMR}\left(400 \mathrm{MHz}, \mathrm{DMSO}-d_{6}\right.$ ): $\delta 3.73$ (s, $3 \mathrm{H}, \mathrm{H}-16)$, 3.75 (s, 3H, H-17), 6.68 (dd, $\left.1 \mathrm{H}, J_{1}=3.6 \mathrm{~Hz}, \mathrm{~J}_{2}=1.6 \mathrm{~Hz}, \mathrm{H}-2\right), 6.89-6.86(\mathrm{~m}, 2 \mathrm{H}, \mathrm{H}-14$ and H-15), 7.08 (brs, 1H, H-11), 7.25 (d, 1H, J=3.2 Hz, H-3), 7.92 (d, 1H, J=0.8 Hz, H-1), 9.58 (brs, 1H, H-6), 9.70 (brs, $1 \mathrm{H}, \mathrm{H}-9), 10.38$ (s, 1H, H-7). ${ }^{13} \mathrm{C}$ NMR (150 MHz, DMSO-d 6 ): $\delta 55.89$ (C-17), 56.05 (C-16), 110.98 (C-11), 111.40 (C-2), 112.32 (C-14), 115.24 (C-3), 118.36 (C-15), 132.65 (C-10), 146.07 (C-1), 146.70 (C-13), 146.87 (C-4), 148.25 (C-12), 158.10 (C-5), 181.44 (C-8). Anal. calc. for $\left(\mathrm{C}_{14} \mathrm{H}_{15} \mathrm{~N}_{3} \mathrm{O}_{4} \mathrm{~S}\right.$ ): C, 52.33; $\mathrm{H}, 4.70 ; \mathrm{N}$, 13.08; S, 9.98\%; found: C, 52.26; $H, 4.61 ; \mathrm{N}, 13.35 ; \mathrm{S}, 9.86 \%$.

\section{1-[(furan-2-carbonyl)]-4-[3,5-(dimethoxy)phenyl]thiosemicarbazide (9)}

White solid. Yield: 65\%; m.p. 203-205 C. FTIR vmax $\left(\mathrm{cm}^{-1}\right)$ : 3654, 3320, 3145 (NH stretching), 2961 (ArCH stretching), 2813 (R-CH), 1689 (C=0), 1264 (C-N), 1057 (C=S). ${ }^{1} \mathrm{H}-\mathrm{NMR}\left(400 \mathrm{MHz}, \mathrm{DMSO}-d_{6}\right): \delta 3.72$ (s, 6H, H-14), 6.31 (brs, 1H, H-2), 6.65 (s, 1H, H-13), 6.79 (brs, 2H, H-11), 7.25 (d, 1H, J=2.4 Hz, H-3), 7.92 (brs, $1 \mathrm{H}, \mathrm{H}-1), 9.69$ (s, 2H, H-6 and H-9), $10.40(\mathrm{~s}, 1 \mathrm{H}, \mathrm{H}-7) .{ }^{13} \mathrm{C} \mathrm{NMR}\left(150 \mathrm{MHz}\right.$, DMSO- $d_{6}$ ): $\delta 55.63$ (C-14), 97.32 (C-13), 104.00 (C-11), 112.34 (C-2), 115.31 (C-3), 141.23 (C-10), 146.13 (C-1), 146.82 (C-4), 158.10 (C-5), 160.15 (C-12), 181.01 (C-8). Anal. calc. for $\left(\mathrm{C}_{14} \mathrm{H}_{15} \mathrm{~N}_{3} \mathrm{O}_{4} \mathrm{~S}\right)$ : C, 52.33; $\mathrm{H}, 4.70 ; \mathrm{N}, 13.08 ; \mathrm{S}, 9.98 \%$; found: $\mathrm{C}$, $52.31 ; \mathrm{H}, 4.76 ; \mathrm{N}, 13.09 ; \mathrm{S}, 9.94 \%$.

\section{1-[(furan-2-carbonyl)]-4-[3,4,5-(trimethoxy)phenyl]thiosemicarbazide (10)}


White solid. Yield: 50\%, m.p. 222-224 ${ }^{\circ} \mathrm{C}$. FTIR vmax $\left(\mathrm{cm}^{-1}\right)$ : 3748, 3241, $3136(\mathrm{NH}$ stretching), 2988 (ArCH stretching), 2842 (R-CH), 1681 (C=0), 1263 (C-N), 1013 (C=S). ${ }^{1} \mathrm{H}-\mathrm{NMR}\left(400 \mathrm{MHz}, \mathrm{DMSO}-d_{6}\right.$ ): $\delta 3.65$ (s, $\left.3 \mathrm{H}, \mathrm{H}_{15}\right), 3.75\left(\mathrm{~s}, 6 \mathrm{H}, \mathrm{H}_{14}\right), 6.68\left(\mathrm{dd}, 1 \mathrm{H}, J_{1}=3.2 \mathrm{~Hz}, J_{2}=1.6 \mathrm{~Hz}, \mathrm{H}_{2}\right), 6.89\left(\mathrm{brs}, 2 \mathrm{H}, \mathrm{H}_{11}\right), 7.26(\mathrm{~d}, 1 \mathrm{H}, \mathrm{J}=3.2$ $\mathrm{Hz}, \mathrm{H}_{3}$ ), 7.92 (brs, $1 \mathrm{H}, \mathrm{H}_{1}$ ), 9.66 (brs, $2 \mathrm{H}, \mathrm{H}_{6}$ and $\mathrm{H}_{9}$ ), 10.38 (brs, $\left.1 \mathrm{H}, \mathrm{H}_{7}\right) .{ }^{13} \mathrm{C}$ NMR (150 MHz, DMSO-d $\left.d_{6}\right): \delta$ $55.25\left(\mathrm{C}_{14}\right), 60.51\left(\mathrm{C}_{15}\right), 103.58\left(\mathrm{C}_{11}\right), 112.35\left(\mathrm{C}_{2}\right), 115.34\left(\mathrm{C}_{3}\right), 135.06\left(\mathrm{C}_{10}\right), 135.38\left(\mathrm{C}_{13}\right), 146.14\left(\mathrm{C}_{1}\right)$, $146.81\left(\mathrm{C}_{4}\right), 152.45\left(\mathrm{C}_{12}\right), 158.16\left(\mathrm{C}_{5}\right), 181.02\left(\mathrm{C}_{8}\right)$. Anal. calc. for $\left(\mathrm{C}_{15} \mathrm{H}_{17} \mathrm{~N}_{3} \mathrm{O}{ }_{5} \mathrm{~S}\right)$ : $\mathrm{C}, 51.27 ; \mathrm{H}, 4.88 ; \mathrm{N}$, 11.96; S: $9.13 \%$; found: $C, 51.23 ; H, 4.84 ; N, 11.90 ;$ S: $9.10 \%$.

\section{1-[(furan-2-carbonyl)]-4-[4-(dimethylamino)phenyl]thiosemicarbazide (11)}

White solid. Yield: $67 \%$; m.p. $214-216^{\circ} \mathrm{C}$. FTIR vmax $\left(\mathrm{cm}^{-1}\right)$ : 3658, 3351, $3132(\mathrm{NH}$ stretching), 2961 (ArCH stretching), 2805 (R-CH), 1683 (C=0), 1263 (C-N), 1056 (C=S). ${ }^{1} \mathrm{H}-\mathrm{NMR}\left(400 \mathrm{MHz}, \mathrm{DMSO}-d_{6}\right): \delta 2.88$ (s, 6H, H-14), 6.67-6.69 (m, 3H, H-2 and H-11), 7.17 (d, 2H, J=8.8 Hz, H-12), 7.24 (d, 1H, J=3.2 Hz, H-3), 7.91 (d, $1 \mathrm{H}, \mathrm{J}=0.8 \mathrm{~Hz}, \mathrm{H}-1), 9.45$ (s, $1 \mathrm{H}, \mathrm{H}-6), 9.59(\mathrm{~s}, 1 \mathrm{H}, \mathrm{H}-9), 10.35(\mathrm{~s}, 1 \mathrm{H}, \mathrm{H}-7) .{ }^{13} \mathrm{C}$ NMR $\left(150 \mathrm{MHz}, \mathrm{DMSO}-d_{6}\right): \delta$ 40.79 (C-14), 112.17 (C-12), 112.26 (C-2), 115.12 (C-3), 127.36 (C-11), 128.77 (C-10), 146.00 (C-1), 146.93 (C-4), 148.69 (C-13), 158.05 (C-5), 181.71 (C-8). Anal. calc. for $\left(\mathrm{C}_{14} \mathrm{H}_{16} \mathrm{~N}_{4} \mathrm{O}_{2} \mathrm{~S}\right.$ ): C, 55.25; $\mathrm{H}, 5.30 ; \mathrm{N}, 18.41$; S, $10.53 \%$; found: C, $55.21 ; \mathrm{H}, 5.28 ; \mathrm{N}, 18.35 ; \mathrm{S}, 10.42 \%$.

\section{1-[(furan-2-carbonyl)]-4-[4-(diethylamino)phenyl]thiosemicarbazide (12)}

White solid. Yield: $62 \%$; m.p. $215-217^{\circ} \mathrm{C}$. FTIR vmax $\left(\mathrm{cm}^{-1}\right): 3672,3346,3203$ (NH stretching), 3114 (ArCH stretching), 2967 (R-CH), 1686 (C=0), 1265 (C-N), 1079 (C=S). ${ }^{1} \mathrm{H}-\mathrm{NMR}\left(400 \mathrm{MHz}, \mathrm{DMSO}-d_{6}\right): \delta 1.08$ (t, 6H, $J=6.8 \mathrm{~Hz}, \mathrm{H}-15), 3.30(\mathrm{q}, 4 \mathrm{H}, \mathrm{H}-14), 6.60(\mathrm{~d}, 2 \mathrm{H}, J=8.8 \mathrm{~Hz}, \mathrm{H}-11), 6.70\left(\mathrm{dd}, 1 \mathrm{H}, J_{7}=3.2 \mathrm{~Hz}, J_{1}=1.6 \mathrm{~Hz}, \mathrm{H}-2\right)$, 7.12 (d, 2H, J=8.4 Hz, H-12), 7.23 (d, 1H, J=3.2 Hz, H-3), 7.90 (brs, 1H, H-1), $9.42(\mathrm{~s}, 1 \mathrm{H}, \mathrm{H}-6), 9.55$ (s, $1 \mathrm{H}, \mathrm{H}-$ 9), $10.34(\mathrm{~s}, 1 \mathrm{H}, \mathrm{H}-7) .{ }^{13} \mathrm{C} \mathrm{NMR}\left(150 \mathrm{MHz}, \mathrm{DMSO}-d_{6}\right): \delta 12.88$ (C-15), 44.16 (C-14), 111.25 (C-2), 112.26 (C12), 115.10 (C-3), 127.63 (C-11), 127.70 (C-10), 145.68 (C-13), 146.00 (C-1), 146.93 (C-4), 158.02 (C-5), 181.55 (C-8). Anal. calc. for $\left(\mathrm{C}_{16} \mathrm{H}_{20} \mathrm{~N}_{4} \mathrm{O}_{2} \mathrm{~S}\right): \mathrm{C}, 57.81 ; \mathrm{H}, 6.06 ; \mathrm{N}, 16.85 ; \mathrm{S}, 9.65 \%$; found: $\mathrm{C}, 57.86 ; \mathrm{H}, 6.03$; N, $16.77 ;$ S, 9.62\%.

\section{General synthetic procedure for compounds 13-24}

A mixture of thiosemicarbazides 1-12 $(0.45 \mathrm{mmol})$ and $2 \mathrm{~N} \mathrm{NaOH}$ solution $(10 \mathrm{~mL})$ was refluxed for $4 \mathrm{~h}$. The reaction mixture was filtered, allowed to cool, and then brought to $\mathrm{pH}$ 5.0-6.0 with a dilute solution of $\mathrm{HCl}$. The crude product was dried, washed with water, and recrystallized from ethanol [31].

\section{5-(2-furyl)-4-phenyl-2,4-dihydro-1,2,4-triazole-3-thione (13) [32]}

Cream solid. Yield: 67\%; m.p. 209-211 ${ }^{\circ} \mathrm{C}$. FTIR vmax ( $\left.\mathrm{cm}^{-1}\right): 3094$ (NH stretching), 2986 (ArCH stretching), 1272 (C-N), 1015 (C=S). ${ }^{1} \mathrm{H}-\mathrm{NMR}\left(400 \mathrm{MHz}, \mathrm{DMSO}-d_{6}\right): \delta 5.89$ (d, $\left.1 \mathrm{H}, \mathrm{J}=3.6 \mathrm{~Hz}, \mathrm{H}-3\right), 6.50$ (dd, $1 \mathrm{H}, J_{7}=3.6$ $\left.\mathrm{Hz}, \mathrm{J}_{2}=1.6 \mathrm{~Hz}, \mathrm{H}-2\right), 7.43-7.45(\mathrm{~m}, 2 \mathrm{H}, \mathrm{H}-12), 7.59-7.61(\mathrm{~m}, 3 \mathrm{H}, \mathrm{H}-11$ and $\mathrm{H}-13), 7.81(\mathrm{~d}, 1 \mathrm{H}, \mathrm{J}=1.2 \mathrm{~Hz}, \mathrm{H}-1)$, 
14.15 (s, $1 \mathrm{H}, \mathrm{H}-7) .{ }^{13} \mathrm{C}$ NMR (100 MHz, DMSO- $\left.d_{6}\right): \delta 112.07$ (C-2), 113.02 (C-3), 129.09 (C-11), 130.40 (C13), 134.81 (C-12), 140.31 (C-10), 143.61 (C-1), 145.74 (C-4), 158.84 (C-5), 169.18 (C-8). Anal. calc. for $\left(\mathrm{C}_{12} \mathrm{H}_{9} \mathrm{~N}_{3} \mathrm{OS}\right): \mathrm{C}, 59.24 ; \mathrm{H}, 3.73 ; \mathrm{N}, 17.27 ; \mathrm{S}, 13.18 \%$; found: $\mathrm{C}, 59.21 ; \mathrm{H}, 3.75 ; \mathrm{N}, 17.12 ; \mathrm{S}, 13.11 \%$.

\section{5-(2-furyl)-4-(4-chlorophenyl)-2,4-dihydro-1,2,4-triazole-3-thione (14) [32]}

White solid. Yield: 52\%; m.p. 224-226 ${ }^{\circ} \mathrm{C}$. FTIR vmax $\left(\mathrm{cm}^{-1}\right)$ : 3092 (NH stretching), 2982 (ArCH stretching), 1265 (C-N), 1091 (C=S), 1024 (C-Cl). ${ }^{1} \mathrm{H}-\mathrm{NMR}\left(400 \mathrm{MHz}, \mathrm{DMSO}-\mathrm{d}_{6}\right.$ ): $\delta 6.11$ (d, $\left.1 \mathrm{H}, \mathrm{J}=3.6 \mathrm{~Hz}, \mathrm{H}-3\right), 6.54$ (dd, $\left.1 \mathrm{H}, J_{1}=3.6, J_{2}=1.2 \mathrm{~Hz}, \mathrm{H}-2\right), 7.51$ (d, 2H, J=8.8 Hz, H-11), 7.67 (d, 2H, J=8.8 Hz, H-12), $7.82(\mathrm{~d}, 1 \mathrm{H}, J=1.2 \mathrm{~Hz}$, $\mathrm{H}-1), 14.02(\mathrm{~s}, 1 \mathrm{H}, \mathrm{H}-6) .{ }^{13} \mathrm{C}$ NMR (100 MHz, DMSO-d 6 ): $\delta 112.25$ (C-2), 113.21 (C-3), 130.13 (C-11), 131.17 (C-12), 133.71 (C-13), 135.12 (C-10), 140.17 (C-1), 143.45 (C-4), 145.89 (C-5), 168.97 (C-8). Anal. calc. for $\left(\mathrm{C}_{12} \mathrm{H}_{8} \mathrm{ClN}_{3} \mathrm{OS}\right)$ : $\mathrm{C}, 51.90 ; \mathrm{H}, 2.90 ; \mathrm{N}, 15.13 ; \mathrm{S}, 11.55 \%$; found: $\mathrm{C}, 51.82 ; \mathrm{H}, 2.81 ; \mathrm{N}, 15.16 ; \mathrm{S}, 11.57 \%$.

5-(2-furyl)-4-(3,5-dichlorophenyl)-2,4-dihydro-1,2,4-triazole-3-thione (15)

Cream solid. Yield: $85 \%$; m.p. $217-219^{\circ} \mathrm{C}$. FTIR vmax ( $\mathrm{cm}^{-1}$ ): 3056 (NH stretching), 2971 (ArCH stretching), 1278 (C-N), 1104 (C=S), 980 (C-Cl). ${ }^{1} \mathrm{H}-\mathrm{NMR}\left(400 \mathrm{MHz}, \mathrm{DMSO}-d_{6}\right): \delta 6.28$ (d, 1H, J=3.6 Hz, H-3), 6.58 (dd, $\left.1 \mathrm{H}, J_{1}=3.6 \mathrm{~Hz}, J_{2}=1.6 \mathrm{~Hz}, \mathrm{H}-2\right), 7.73(\mathrm{~d}, 1 \mathrm{H}, J=1.6 \mathrm{~Hz}, \mathrm{H}-1), 7.83(\mathrm{brs}, 1 \mathrm{H}, \mathrm{H}-13), 7.87-7.90(\mathrm{~m}, 2 \mathrm{H}, \mathrm{H}-11)$, $14.22(\mathrm{~s}, 1 \mathrm{H}, \mathrm{H}-7) .{ }^{13} \mathrm{C}$ NMR $\left(100 \mathrm{MHz}, \mathrm{DMSO}-d_{6}\right): \delta 112.38$ (C-2), 113.30 (C-3), 128.71 (C-13), 134.93 (C11), 137.05 (C-12), 140.09 (C-10), 143.20 (C-1), 145.97 (C-4), 154.28 (C-5), 168.86 (C-8). Anal. calc. for $\left(\mathrm{C}_{12} \mathrm{H}_{7} \mathrm{Cl}_{2} \mathrm{~N}_{3} \mathrm{OS}\right): \mathrm{C}, 46.17 ; \mathrm{H}, 2.26 ; \mathrm{N}, 13.46 ; \mathrm{S} .10,27 \%$; found: $\mathrm{C}, 46.23 ; \mathrm{H}, 2.34 ; \mathrm{N}, 13.35 ; \mathrm{S}, 10.29 \%$.

\section{5-(2-furyl)-4-(4-chloro-3-(trifluoromethyl)phenyl)-2,4-dihydro-1,2,4-triazole-3-thione (16)}

White solid. Yield: $72 \%$; m.p. 229-231 ${ }^{\circ} \mathrm{C}$. FTIR vmax $\left(\mathrm{cm}^{-1}\right)$ : 3080 (NH stretching), 2971 (ArCH stretching), 1275(C-N), 1131 (C-F), 1073 (C-Cl), 1071 (C=S). ${ }^{1} \mathrm{H}-\mathrm{NMR}$ (400 MHz, DMSO- $\left.d_{6}\right): \delta 6.30$ (d, 1H, J=3.6 Hz, H3), $6.56\left(\mathrm{dd}, 1 \mathrm{H}, J_{1}=3.6, J_{2}=1.6 \mathrm{~Hz}, \mathrm{H}-2\right), 7.78-8.01(\mathrm{~m}, 2 \mathrm{H}, \mathrm{H}-11$ and $\mathrm{H}-15), 7.97(\mathrm{~d}, 1 \mathrm{H}, \mathrm{J}=8.4 \mathrm{~Hz}, \mathrm{H}-14)$, 8.14 (d, $1 \mathrm{H}, J=2.4 \mathrm{~Hz}, \mathrm{H}-1), 14.21$ (brs, $1 \mathrm{H}, \mathrm{H}-7) .{ }^{13} \mathrm{C} \mathrm{NMR}\left(100 \mathrm{MHz}, \mathrm{DMSO}-d_{6}\right): \delta 112.35$ (C-2), 113.31 (C3), 124.17 (C-11), 129.31 (C-13), 132.70 (C-16), 134.33 (C-15), 135.27 (C-12), 140.19 (C-14), 142.34 (C-10), 143.27 (C-1), 145.91 (C-4), 155.48 (C-5), 168.90 (C-8). Anal. calc. for $\left(\mathrm{C}_{13} \mathrm{H}_{7} \mathrm{ClF}_{3} \mathrm{~N}_{3} \mathrm{OS}\right.$ ): $\mathrm{C}, 45.16 ; \mathrm{H}, 2.04 ; \mathrm{N}$, $12.15 ; \mathrm{S}, 9.27 \%$; found: $\mathrm{C}, 45.07 ; \mathrm{H}, 2.11 ; \mathrm{N}, 12.01 ; \mathrm{S}, 9.30 \%$.

5-(2-furyl)-4-(5-chloro-2-methoxyphenyl)-2,4-dihydro-1,2,4-triazole-3-thione (17) [32]

White solid. Yield: 88\%; m.p. 216-218 ${ }^{\circ} \mathrm{C}$. FTIR vmax $\left(\mathrm{cm}^{-1}\right)$ : 3094 (NH stretching), 2971 (ArCH stretching), 2773 (R-CH), 1281 (C-N), 1028 (C=S), 980 (C-Cl). ${ }^{1} \mathrm{H}-\mathrm{NMR}$ (400 MHz, DMSO-d $_{6}$ ): $\delta 3.69$ (s, 3H, H-16), 6.14 (d, $1 \mathrm{H}, J=3.6 \mathrm{~Hz}, \mathrm{H}-3), 6.54$ (dd, $\left.1 \mathrm{H}, J_{1}=3.6, J_{2}=1.6 \mathrm{~Hz}, \mathrm{H}-2\right), 7.30$ (d, 1H, J=8.8 Hz, H-13), 7.61-7.66 (m, 2H, $\mathrm{H}-12$ and $\mathrm{H}-15), 7.81$ (d, $1 \mathrm{H}, \mathrm{J}=1.2 \mathrm{~Hz}, \mathrm{H}-1), 14.02$ (brs, $1 \mathrm{H}, \mathrm{H}-7) .{ }^{13} \mathrm{C}$ NMR $(150 \mathrm{MHz}$, DMSO-d 6 ): $\delta 56.96$ (C-16), 112.10 (C-2), 112.21 (C-3), 115.16 (C-12), 124.39 (C-15), 124.58 (C-13), 130.56 (C-10), 131.87 (C- 
14), 140.49 (C-11), 143.70 (C-1), 145.69 (C-4), 154.63 (C-5), 169.22 (C-8). Anal. calc. for $\left(\mathrm{C}_{13} \mathrm{H}_{10} \mathrm{ClN}_{3} \mathrm{O}_{2} \mathrm{~S}\right)$ : C, $50.73 ; H, 3.28 ; \mathrm{N}, 13.65 ; \mathrm{S}, 10.42 \%$; found: C, $50.59 ; \mathrm{H}, 3.23 ; \mathrm{N}, 13.58 ; \mathrm{S}, 10.29 \%$.

5-(2-furyl)-4-(2,4-dimethoxyphenyl)-2,4-dihydro-1,2,4-triazole-3-thione (18)

Brown solid. Yield: 65\%; m.p. 205-207 C. FTIR vmax ( $\left.\mathrm{cm}^{-1}\right)$ : 3286 (NH stretching), 2918 (ArCH stretching), 1211 (C-N), 1048 (C=S). ${ }^{1} \mathrm{H}-\mathrm{NMR}\left(400 \mathrm{MHz}, \mathrm{DMSO}-\mathrm{d}_{6}\right): 3.67$ (s, 3H, H-17), 3.86 (s, 3H, H-16), 5.96 (brs, $1 \mathrm{H}$, $\mathrm{H}-3$ ), 6.51 (brs, 1H, H-2), 6.70 (d, 1H, J=7.2 Hz, H-14), 6.79 (brs, 1H, H-12), 7.29 (d, 1H, J=8.0 Hz, H-15), 7.81 (brs, 1H, H-1), $14.02(\mathrm{~s}, 1 \mathrm{H}, \mathrm{H}-7) .{ }^{13} \mathrm{C}$ NMR $\left(150 \mathrm{MHz}\right.$, DMSO- $\left.d_{6}\right): \delta 56.10$ (C-16), 56.54 (C-17), 100.20 (C12), 106.14 (C-14), 111.97 (C-2), 112.19 (C-3), 115.83 (C-15), 131.22 (C-10), 144.21 (C-1), 145.67 (C-4), 156.36 (C-11), 162.28 (C-5), 169.46 (C-8). Anal. calc. for $\left(\mathrm{C}_{14} \mathrm{H}_{13} \mathrm{~N}_{3} \mathrm{O}_{3} \mathrm{~S}\right)$ : C, 55.43; $\mathrm{H}, 4.32 ; \mathrm{N}, 13.85 ; \mathrm{S}$, 10.57\%; found: $C, 55.40 ; \mathrm{H}, 4.31 ; \mathrm{N}, 13.76 ; \mathrm{S}, 10.55 \%$.

5-(2-furyl)-4-(2,5-dimethoxyphenyl)-2,4-dihydro-1,2,4-triazole-3-thione (19)

Cream solid. Yield: 85\%; m.p. 210-212 ${ }^{\circ} \mathrm{C}$. FTIR vmax ( $\mathrm{cm}^{-1}$ ): 3082 (NH stretching), 2913 (ArCH stretching), 2776 (R-CH), 1224 (C-N), 1043 (C=S). ${ }^{1} \mathrm{H}-\mathrm{NMR}\left(400 \mathrm{MHz}, \mathrm{DMSO}-d_{6}\right): \delta 3.61$ (s, 3H, H-16), 3.75 (s, 3H, H17), $5.94(\mathrm{~d}, 1 \mathrm{H}, \mathrm{J}=3.6 \mathrm{~Hz}, \mathrm{H}-3), 6.50(\mathrm{q}, 1 \mathrm{H}, \mathrm{H}-2), 7.03$ (d, 1H, J=2.8 Hz, H-15), 7.13 (d, 1H, J=9.2, H-13), 7.19 (d, $1 \mathrm{H}, \mathrm{J}=9.2 \mathrm{~Hz}, \mathrm{H}-12), 7.79$ (brs, $1 \mathrm{H}, \mathrm{H}-1), 14.00(\mathrm{~s}, 1 \mathrm{H}, \mathrm{H}-7) .{ }^{13} \mathrm{C}$ NMR (150 MHz, DMSO-d $\left.)_{6}\right): \delta 56.30$ (C-17), 56.97 (C-16), 111.51 (C-2), 112.07 (C-3), 114.67 (C-15), 116.58 (C-13), 116.87 (C-12), 124.17 (C-10), 140.92 (C-11), 149.63 (C-14), 143.91 (C-1), 145.32 (C-4), 153.76 (C-5), 169.27 (C-8). Anal. calc. for $\left(\mathrm{C}_{14} \mathrm{H}_{13} \mathrm{~N}_{3} \mathrm{O}_{3} \mathrm{~S}\right)$ : $\mathrm{C}, 55.43 ; \mathrm{H}, 4.32 ; \mathrm{N}, 13.85 ; \mathrm{S}, 10.57 \%$; found: $\mathrm{C}, 55.40 ; \mathrm{H}, 4.39 ; \mathrm{N}, 13.75 ; \mathrm{S}, 10.36 \%$.

5-(2-furyl)-4-(3,4-dimethoxyphenyl)-2,4-dihydro-1,2,4-triazole-3-thione (20)

Cream solid. Yield: 93\%; m.p. 206-208 ${ }^{\circ} \mathrm{C}$. FTIR vmax ( $\left.\mathrm{cm}^{-1}\right)$ : 3276 (NH stretching), 2974 (ArCH stretching), 2770 (R-CH), 1221 (C-N), 1043 (C=S). ${ }^{1} \mathrm{H}-\mathrm{NMR}\left(400 \mathrm{MHz}, \mathrm{DMSO}-d_{6}\right): \delta 3.72$ (s, 3H, H-17), 3.85 (s, 3H, H16), $5.92(\mathrm{~d}, 1 \mathrm{H}, \mathrm{J}=2.8 \mathrm{~Hz}, \mathrm{H}-3), 6.51$ (dd, 1H, $\left.J_{1}=3.2, J_{2}=2.8 \mathrm{H}-2\right), 6.95$ (d, 1H, J=8.4, H-14), 7.07-7.13 (m, $2 \mathrm{H}, \mathrm{H}-11$ and $\mathrm{H}-15), 7.82$ (brs, $1 \mathrm{H}, \mathrm{H}-1), 14.05$ (brs, $1 \mathrm{H}, \mathrm{H}-7) .{ }^{13} \mathrm{C}$ NMR $\left(150 \mathrm{MHz}, \mathrm{DMSO}-d_{6}\right): \delta 56.28(\mathrm{C}-$ 17), 56.50 (C-16), 112.43 (C-2), 112.11 (C-11), 112.91 (C-3), 113.06 (C-14), 121.48 (C-15), 127.34 (C-10), 140.51 (C-13), 149.74 (C-12), 143.92 (C-1), 145.57 (C-4), 150.35 (C-5), 169.37 (C-8). Anal. calc. for $\left(\mathrm{C}_{14} \mathrm{H}_{13} \mathrm{~N}_{3} \mathrm{O}{ }_{3} \mathrm{~S}\right): \mathrm{C}, 55.43 ; \mathrm{H}, 4.32 ; \mathrm{N}, 13.85 ; \mathrm{S}, 10.57 \%$; found: $\mathrm{C}, 55.42 ; \mathrm{H}, 4.30 ; \mathrm{N}, 13.81 ; \mathrm{S}, 10.48 \%$.

5-(2-furyl)-4-(3,5-dimethoxyphenyl)-2,4-dihydro-1,2,4-triazole-3-thione (21)

Yellow solid. Yield: 91\%; m.p. 209-211 ${ }^{\circ} \mathrm{C}$. FTIR vmax $\left(\mathrm{cm}^{-1}\right): 3218$ (NH stretching), 2970 (ArCH stretching), 2771 (R-CH), 1207 (C-N), 1159 (C=S). ${ }^{1} \mathrm{H}-\mathrm{NMR}\left(400 \mathrm{MHz}, \mathrm{DMSO}-d_{6}\right): \delta 3.76$ (s, 6H, H-14), 6.03 (d, 1H, $J=3.2 \mathrm{~Hz}, \mathrm{H}-3), 6.53\left(\mathrm{dd}, 1 \mathrm{H}, J_{1}=3.2, J_{2}=1.6 \mathrm{~Hz}, \mathrm{H}-2\right), 6.63(\mathrm{~d}, 2 \mathrm{H}, \mathrm{J}=2.0 \mathrm{~Hz}, \mathrm{H}-11), 6.71(\mathrm{~d}, 1 \mathrm{H}, J=2.0, \mathrm{H}-13)$, $7.83(\mathrm{~d}, 1 \mathrm{H}, J=1.6 \mathrm{~Hz}, \mathrm{H}-1), 13.97$ (brs, $1 \mathrm{H}, \mathrm{H}-7) .{ }^{13} \mathrm{C}$ NMR (100 MHz, DMSO- $\left.d_{6}\right): \delta 56.15$ (C-14), 102.07 (C13), 112.21 (C-2), 112.88 (C-3), 107.54 (C-11), 136.38 (C-10), 140.29 (C-1), 143.51 (C-4), 145.69 (C-5), 
161.35 (C-12), 168.82 (C-8). Anal. calc. for $\left(\mathrm{C}_{14} \mathrm{H}_{13} \mathrm{~N}_{3} \mathrm{O}_{3} \mathrm{~S}\right)$ : C, 55.43; $\mathrm{H}, 4.32 ; \mathrm{N}, 13.85 ; \mathrm{S}, 10.57 \%$; found: $\mathrm{C}$, $55.40 ; \mathrm{H}, 4.28 ; \mathrm{N}, 13.81 ; \mathrm{S}, 10.51 \%$.

5-(2-furyl)-4-(3,4,5-trimethoxyphenyl)-2,4-dihydro-1,2,4-triazole-3-thione (22)

Cream solid. Yield: $47 \%$; m.p. 228-230 ${ }^{\circ} \mathrm{C}$. FTIR vmax $\left(\mathrm{cm}^{-1}\right)$ : 3242 (NH stretching), 2972 (ArCH stretching), 2769 (R-CH), 1235 (C-N), 1128 (C=S). ${ }^{1} \mathrm{H}-\mathrm{NMR}\left(400 \mathrm{MHz}, \mathrm{DMSO}-d_{6}\right): \delta 3.74(\mathrm{~s}, 6 \mathrm{H}, \mathrm{H}-14), 3.77(\mathrm{~s}, 3 \mathrm{H}, \mathrm{H}-$ 15), $5.98(\mathrm{~d}, 1 \mathrm{H}, \mathrm{J}=3.6 \mathrm{~Hz}, \mathrm{H}-3), 6.54\left(\mathrm{dd}, 1 \mathrm{H}, J_{7}=3.6 \mathrm{~Hz}, J_{2}=1.6 \mathrm{~Hz}, \mathrm{H}-2\right), 6.84$ (brs, 2H, H-11), $7.92(\mathrm{~d}, 1 \mathrm{H}$, $J=1.2 \mathrm{~Hz}, \mathrm{H}-1), 14.00$ (s, 1H, H-7). ${ }^{13} \mathrm{C}$ NMR (100 MHz, DMSO- $d_{6}$ ): $\delta 56.81$ (C-15), 60.69 (C-14), 112.28 (C2), 112.95 (C-3), 107.06 (C-11), 130.18 (C-10), 138.93 (C-13), 140.31 (C-1), 143.37 (C-4), 145.67 (C-12), 153.80 (C-5), 168.99 (C-8). Anal. calc. for $\left(\mathrm{C}_{15} \mathrm{H}_{15} \mathrm{~N}_{3} \mathrm{O}_{4} \mathrm{~S}\right)$ : C, 54.04; $\mathrm{H}, 4.54 ; \mathrm{N}, 12.60 ; \mathrm{S}, 9.62 \%$; found: $\mathrm{C}$, $54.07 ; \mathrm{H}, 4.56 ; \mathrm{N}, 12.57 ; \mathrm{S}, 9.58 \%$.

5-(2-furyl)-4-(4-(dimethylamino)phenyl)-2,4-dihydro-1,2,4-triazole-3-thione (23)

Cream solid. Yield: 89\%; m.p. 221-223ํ․ FTIR vmax ( $\mathrm{cm}^{-1}$ ): 3196 (NH stretching), 2972 (ArCH stretching), 2770 (R-CH), 1327 (C-N), 1230 (C=S). ${ }^{1} \mathrm{H}-\mathrm{NMR}\left(400 \mathrm{MHz}, \mathrm{DMSO}-d_{6}\right): \delta 3.00$ (s, 6H, H-14), 5.86 (d, 1H, J=3.2 Hz, H-3), 6.51 (dd, 1H, J $\left.=3.6, J_{2}=3.6 \mathrm{~Hz}, \mathrm{H}-2\right), 6.82(\mathrm{~d}, 2 \mathrm{H}, \mathrm{J}=8.8 \mathrm{~Hz}, \mathrm{H}-11), 7.15(\mathrm{~d}, 2 \mathrm{H}, \mathrm{J}=8.8 \mathrm{~Hz}, \mathrm{H}-$ 12), 7.83 (brs, $1 \mathrm{H}, \mathrm{H}-1), 14.02$ (brs, $1 \mathrm{H}, \mathrm{H}-7) .{ }^{13} \mathrm{C}$ NMR (100 MHz, DMSO- $\left.d_{6}\right): \delta 40.72$ (C-14), 112.05 (C-2), 112.56 (C-3), 112.81 (C-12), 122.59 (C-11), 129.32 (C-10), 140.64 (C-13), 144.14 (C-1), 145.56 (C-4), 151.32 (C-5), 169.51 (C-8). Anal. calc. for $\left(\mathrm{C}_{14} \mathrm{H}_{14} \mathrm{~N}_{4} \mathrm{OS}\right)$ : C, 58.72; $\mathrm{H}, 4.93 ; \mathrm{N}, 19.57 ; \mathrm{S}, 11.20 \%$; found: $\mathrm{C}$, $58.66 ; \mathrm{H}, 4.95 ; \mathrm{N}, 19.48 ; \mathrm{S}, 11.14 \%$.

5-(2-furyl)-4-(4-(diethylamino)phenyl)-2,4-dihydro-1,2,4-triazole-3-thione (24)

Cream solid. Yield: $80 \%$; m.p. $219-220^{\circ} \mathrm{C}$. FTIR vmax ( $\left.\mathrm{cm}^{-1}\right)$ : 3076 (NH stretching), 2977 (ArCH stretching), 2767 (R-CH), 1270 (C-N), 1190 (C=S). ${ }^{1} \mathrm{H}-\mathrm{NMR}\left(400 \mathrm{MHz}, \mathrm{DMSO}-d_{6}\right): \delta 1.15$ (t, 6H, J=6.8 Hz, H-15), 3.41 (q, $4 \mathrm{H}, \mathrm{H}-14), 5.87(\mathrm{~d}, 1 \mathrm{H}, \mathrm{J}=3.6 \mathrm{~Hz}, \mathrm{H}-3), 6.53\left(\mathrm{dd}, 1 \mathrm{H}, J_{1}=3.6, J_{2}=1.6 \mathrm{~Hz}, \mathrm{H}-2\right), 6.77$ (d, 2H, J=9.2 Hz, H-11), $7.12(\mathrm{~d}, 2 \mathrm{H}, \mathrm{J}=9.2 \mathrm{~Hz}, \mathrm{H}-12), 7.85$ (d, $1 \mathrm{H}, \mathrm{J}=0.8 \mathrm{~Hz}, \mathrm{H}-1), 14.02(\mathrm{~s}, 1 \mathrm{H}, \mathrm{H}-7) .{ }^{13} \mathrm{C}$ NMR (100 MHz, DMSO-d $)_{6}$ : $\delta 12.81$ (C-15), 44.22 (C-14), 111.82 (C-2), 112.07 (C-3), 112.84 (C-12), 121.58 (C-11), 129.55 (C-10), 140.64 (C-13), 144.25 (C-1), 145.56 (C-4), 148.73 (C-5), 169.57 (C-8). Anal. calc. for $\left(\mathrm{C}_{16} \mathrm{H}_{18} \mathrm{~N}_{4} \mathrm{OS}\right)$ : C, 61.12; $H, 5.77 ; \mathrm{N}, 17.82 ; \mathrm{S}, 10.20 \%$; found: $\mathrm{C}, 61.10 ; \mathrm{H}, 5.65 ; \mathrm{N}, 17.71 ; \mathrm{S}, 10.24 \%$.

\section{Biological studies}

\section{Antiproliferative activity assay}

The antiproliferative assessment of the compounds was conducted on HeLa (cervical) cells using the BrdU ELISA [33-36]. The results of this in vitro investigation were given as means \pm SEM of six parallel measurements $(p<0.01) . \mathrm{IC}_{50}$ values were calculated using the online software $\mathrm{ED}_{50}$ plus v1.0. 
Solutions of thiosemicarbazides 1-12 and 1,2,4-triazole 23-24 were prepared at four different concentrations $(25,50,100,200 \mu \mathrm{M})$ in DMSO. Activity was compared using control (DMSO) and antioxidant standards (BHA and a-TOC). The results were presented as $50 \%$ inhibition versus concentration $\left(\mathrm{IC}_{50}\right)$ for the ABTS ${ }^{*+}$ scavenging activity, $\beta$-carotene-linoleic acid, and DPPH ${ }^{\circ}$ assays, while in the CUPRAC assay the results were expressed as 0.5 absorbance versus concentration $\left(A_{0.5}\right)$. As given in the literature, $A B T S^{-+}$scavenging activity [37], lipid peroxidation inhibitory activity ( $\beta$-carotenelinoleic acid assay) [38], cupric reducing antioxidant capacity (CUPRAC assay) [39], and DPPH radical scavenging activity [40] assays were used to determine the antioxidant activity of the thiosemicarbazides 1-12 and 1,2,4-triazoles 23-24.

\section{In silico ADME prediction}

Computational studies of the synthesized compounds 1-24 were performed to predict molecular properties using the SwissADME online server [41]. The molecular volume (Mv), molecular weight (Mw), logarithm of partition coefficient (milog P), number of hydrogen-bond donors (HBDs), number of hydrogen-bond acceptors (HBAs), topological polar surface area (TPSA), number of rotatable bonds (Nrotbs), and Lipinski's rule of five of the synthesized compounds were determined.

\section{Statistical analysis}

The antiproliferative and antioxidant activity data were given as the means of six and three parallel measurements, respectively. All biological activity assays were carried out at four different concentrations, and the results were presented as $\mathrm{IC}_{50}$ values. The data were recorded as mean $\pm \mathrm{SEM}$ (standard error of the mean); $p<0.01$.

\section{Declarations}

\section{Acknowledgements}

The Muğla Sıtkı Koçman University Research Fund was acknowledged for the support through the research project (Project number: 19/084/09/1/2). The author would like to thank Prof. Dr. Ayşe Şahin Yağlıoğlu for her help in determining the antiproliferative activity against the HeLa cell line.

\section{Conflict of Interest}

The authors declare that they have no conflicts of interest to disclose.

\section{References}

1. Husain A, Rashid M, Mishra R, Parveen S, Soo SD, Kumar D. Benzimidazole bearing oxadiazole and triazolo-thiadiazoles nucleus: design and synthesis as anticancer agents. Bioorg. Med. Chem. Lett. 
2012;22:5438-44. https://doi.org/10.1016/j.bmcl.2012.07.038

2. Mirza AZ, Althagafi II, Shamshad H. Role of PPAR receptor in different diseases and their ligands: Physiological importance and clinical implications. Eur. J. Med. Chem. 2019;15:502-13. https://doi.org/10.1016/j.ejmech.2019.01.067

3. Bhatt AN, Mathur R, Farooque A, Verma A, Dwarakanath BS. Cancer biomarkers - current perspectives. Indian J. Med. Res. 2010;132:129-49.

4. Rashid M, Husain A, Mishra R. Synthesis of benzimidazoles bearing oxadiazole nucleus as anticancer agents. Eur. J. Med. Chem. 2012;54:855-66. https://doi.org/10.1016/j.ejmech.2012.04.027

5. Scherer WF, Syverton JT, Gey GO. Studies on the propagation in vitro of poliomyelitis viruses. IV. Viral multiplication in a stable strain of human malignant epithelial cells (strain HeLa) derived from an epidermoid carcinoma of the cervix J. Exp. Med. 1953;97:695-710. https://doi.org/10.1084/jem.97.5.695

6. Bray F, Ferlay J, Soerjomataram I, Siegel RL, Torre LA, Jemal A. Global cancer statistics 2018: GLOBOCAN estimates of incidence and mortality worldwide for 36 cancers in 185 countries. CA Cancer J. Clin. 2018;68:394-424. https://doi.org/10.3322/caac.21492

7. DuPont NC, Monk BJ. Chemotherapy in the management of cervical carcinoma. Clin. Adv. Hematol. Oncol. 2006;4:279-86.

8. Niu Y, Yan C. The Effect of fullerenol combined with cisplatin on the proliferation of cervical cancer HeLa cells. J. Cancer Therapy. 2016;7:232-8. https://doi.org/10.4236/jct.2016.73024

9. Benard VB, Watson M, Castle PE, Saraiya M. Cervical carcinoma rates among young females in the United States. Obstet Gynecol. 2012;120:1117-23.

10. Grigalius I, Petrikaite V. Relationship between antioxidant and anticancer activity of trihydroxyflavones. Molecules. 2017;22:1-12. https://doi.org/10.3390/molecules22122169

11. Bandgar BP, Gawande SS, Bodade RG, Totre JV, Khobragade CN. Synthesis and biological evaluation of simple methoxylated chalcones as anticancer, anti-inflammatory and antioxidant agents. Bioorg. Med. Chem. 2018;18:1364-70. https://doi.org/10.1016/j.bmc.2009.11.066

12. Liou GY, Storz P. Reactive oxygen species in cancer. Free Radic. Res. 2010;44:479-96. https://doi.org/10.3109/10715761003667554

13. Pourahmad J, Salimi A, Seydi E. Free Radicals and Diseases. Intech; 2016

14. Uttara B, Singh AV, Zamboni P, Mahajan RT. Oxidative stress and neurodegenerative diseases: a review of upstream and downstream antioxidant therapeutic options. Curr. Neuropharmacol. 2009;7:65-74. https://doi.org/10.2174/157015909787602823

15. Perrone S, Santacroce A, Longini M, Proietti F, Bazzini F, Buonocore G. The free radical diseases of prematurity: from cellular mechanisms to bedside. Oxid. Med. Cell. Longev. 2018;2018:1-14. https://doi.org/10.1155/2018/7483062 
16. Bendary E, Francis RR, Ali HMG, Sarwat MI, El Hady S. Antioxidant and structure-activity relationships (SARs) of some phenolic and anilines compounds. Annal. Agricul. Sci. 2013;58:173-181. https://doi.org/10.1016/j.aoas.2013.07.002

17. Arora P, Arora V, Lamba HS, Wadhwa D. Importance of heterocyclic chemistry: a review. Int. J. Pharm. Sci. Res. 2012;3:2947-54. https://doi.org/10.13040/IJPSR.0975-8232.3(9).2947-54

18. Pearce $S$. The importance of heterocyclic compounds in anti-cancer drug design. Drug Discovery World. 2017;18:66-70.

19. Verma A, Pandeya SN, Sinha S. Synthesis and biological activities of furan derivatives. Int. J. Res. Ayurveda Pharm. 2011;2:1110-6.

20. Kotan G, Yüksek H. Theoretical and spectroscopic studies of 1-(morpholine-4-yl-methyl)-3-benzyl-4(4-isopropylbenzylidenamino)-4,5-dihydro-1H-1,2,4-triazol-5-one molecule. J. Turk. Chem. Soc. Sect. A. 2016;3:381-92. https://doi.org/10.18596/jotcsa.08773

21. Bladin JA. Ueber von Dicyanphenylhydrazin abgeleitete verbindungen. Ber. dtsch. chem. Ges. 1885;18:1544-51. https://doi.org/ 10.1002/cber.188501801335

22. Bladin JA. Ueber Verbindungen, welche sich vom dicyanphenylhydrazin ableiten. III. Ber. dtsch. chem. Ges. 1886;19:2598-604. https://doi.org/10.1002/cber.188601902220

23. Potts KT. The Chemistry of 1, 2, 4-Triazoles. Chem. Rev. 1960;61:87-127.

24. Demir Kanmazalp S, Başaran E, Karaküçük-lyidoğan A, Oruç-Emre EE, Dege N. X-ray structures and spectroscopic properties of chiral thiosemicarbazides as studied by computational calculations. Phosphorus, Sulfur, and Silicon Relat. Elem. 2017;192: 856-865. https://doi.org/10.1080/10426507.2017.1289380

25. Başaran E, Sıcak Y, Soğukömeroğulları HG, Karaküçük-lyidoğan A, Oruç-Emre EE, Sönmez M, Öztürk $M$. Synthesis of novel chiral metal complexes derived from chiral thiosemicarbazide ligands as potential antioxidant agents. Chirality, 2019;31:434-44. https://doi.org/10.1002/chir.23069

26. Oruç EE, Kaymakçıoğlu-Koçyiğit B, Oral B, Altuntaş-Toklu HZ, Kabasakal L, Rollas S. Synthesis of some novel azo derivatives of 3,5-dimethly-1-(2-hydroxyethyl)pyrazole as potent analgesic agents. Arch. Pharm. Chem. Life Sci. 2006;339:267-72. https://doi.org/10.1002/ardp.200500202

27. Demir Kanmazalp S, Başaran E, Karaküçük-lyidoğan A, Oruç-Emre EE, Dege N, Şen F. Synthesis, characterization, spectroscopy, X-ray structure and gaussian hybrid computational investigation of (-)-(S)-1-[2-(benzenesulfonamido)-3-phenylpropanoyl]-4-[(4-methyl)phenyl]thiosemicarbazide. Phosphorus, Sulfur, and Silicon Relat. Elem. 2018;193:675-84. https://doi.org/10.1080/10426507.2018.1488713

28. Mishra S, Dahima R. In-vitro ADME studies of TUG-891, a GPR-120 inhibitor using Swiss ADME predictor. J. Drug Del. Therapy. 2019;9:366-9. https://dx.doi.org/10.22270/jddt.v9i2-s.2710

29. Daina A, Zoete V. A BOILED-Egg to predict gastrointestinal absorption and brain penetration of small molecules. Chem. Med. Chem. 2016;11:1117-21. https://doi.org/10.1002/cmdc.201600182

30. Hossain MM, Shaha SK, Aziz F. Antioxidant potential study of some synthesized $N$-heterocycles. Bangladesh Med. Res. Counc. Bull. 2009;35:49-52. https://doi.org/10.3329/bmrcb.v35i2.2564 
31. Başaran E, Karaküçük-Iyidoğan A, Schols D, Oruç-Emre EE. Synthesis of novel chiral sulfonamidebearing 1,2,4-triazole-3-thione analogs derived from $\mathrm{D}$ - and L-phenylalanine esters as potential antiinfluenza agents. Chirality. 2016;28:495-513. https://doi.org/10.1002/chir.22607

32. Li XZ, Si ZX, Synthesis and biological activities of 3-(2-furyl)-4-aryl-1, 2, 4-triazole-5-thiones. Chinese Chem. Lett. 2002;13:129-32.

33. Erenler R, Şen Ö, Akşit H, Demirtaş I, Şahin-Yağlıoğlu A, Elmastaş M, Telci İ. Isolation and identification of chemical constituents from Origanum majorana and investigation of antiproliferative and antioxidant activities. J. Sci. Food. Agric. 2016;96: 822-36. https://doi.org/10.1002/jsfa.7155

34. Kasımoğulları R, Duran H, Şahin-Yağlıoğlu A, Mert S, Demirtaş i. Design, synthesis, characterization, and antiproliferative activity of novel pyrazole-3-carboxylic acid derivatives. Monatshefte. Für Chemie. 2015;146:1743-9. https://doi.org/10.1007/s00706-015-1450-7

35. Demirtaş İ, Geçibeşler IH, Şahin-Yağlıoğlu A. Antiproliferative activities of isolated flavone glycosides and fatty acids from Stachys byzantina. Phytochem. Lett. 2013, 6, 209-214. https://doi.org/10.1016/j.phytol.2013.02.001

36. Gürdere MB, Kamo E, Budak Y, Şahin-Yağlıoğlu A, Ceylan M. Synthesis and anticancer and cytotoxic effects of novel 1,4-phenylene-bis- $N$-thiocarbamoylpyrazole and 1,4-phenylene-bis-pyrazolylthiazole derivatives. Turk. J. Chem. 2017;41:179-89. https://doi.org/10.3906/kim-1604-84

37. Re R, Pellegrini N, Proteggente A, Pannala A, Yang M, Rice-Evans C. Antioxidant activity applying an improved ABTS radical cation decolorization assay. Free Radic. Biol. Med. 1999;26:1231-7. https://doi.org/10.1016/s0891-5849(98)00315-3

38. Miller HE. A simplified method for the evaluation of antioxidants. J. Am. Oil Chem. Soc. 1971;48:91.

39. Apak R, Güçlü K, Özyürek M, Karademir SE. Novel total antioxidant capacity index for dietary polyphenols and vitamins $\mathrm{C}$ and $\mathrm{E}$, using their cupricion reducing capability in the presence of neocuproine: CUPRAC method. J. Agric. Food Chem. 2004;52:7970-81. https://doi.org/10.1021/jf048741x

40. Blois MS. Antioxidant Determinations by the use of a stable free radical. Nature. 1958;181:11991200.

41. http://www.swissadme.ch/, (accessed on 10 October 2020)

\section{Tables}


Table 1

$\mathrm{IC}_{50}$ values against HeLa cancer cells of synthesized compounds 1-24

\begin{tabular}{|c|c|c|c|}
\hline \multicolumn{2}{|c|}{ Thiosemicarbazide derivatives } & \multicolumn{2}{|c|}{ 1,2,4-Triazole derivatives } \\
\hline Compounds & $\mathrm{IC}_{50}(\mu \mathrm{M})$ & Compounds & $\mathrm{IC}_{50}(\mu \mathrm{M})$ \\
\hline 1 & $76.93 \pm 0.51$ & 13 & $42.50 \pm 0.81$ \\
\hline 2 & $74.64 \pm 0.19$ & 14 & $47.87 \pm 0.74$ \\
\hline 3 & $19.83 \pm 0.42$ & 15 & $8.81 \pm 0.28$ \\
\hline 4 & $62.73 \pm 0.25$ & 16 & $30.54 \pm 0.65$ \\
\hline 5 & $86.37 \pm 0.33$ & 17 & $45.07 \pm 0.68$ \\
\hline 6 & $61.10 \pm 0.84$ & 18 & $27.31 \pm 0.59$ \\
\hline 7 & $75.16 \pm 0.31$ & 19 & $48.87 \pm 0.27$ \\
\hline 8 & $69.69 \pm 0.96$ & 20 & $44.08 \pm 0.66$ \\
\hline 9 & $97.62 \pm 0.55$ & 21 & $51.44 \pm 0.40$ \\
\hline 10 & $89.57 \pm 0.73$ & 22 & $42.67 \pm 0.67$ \\
\hline 11 & $52.32 \pm 0.61$ & 23 & $53.55 \pm 0.38$ \\
\hline 12 & $68.17 \pm 0.27$ & 24 & $49.12 \pm 0.16$ \\
\hline 5-FU & $4.93 \pm 0.24$ & 5-FU & $4.93 \pm 0.24$ \\
\hline
\end{tabular}


Table 2

Antioxidant activity results of thiosemicarbazide 1-12 and 1,2,4-triazole derivatives 13-24

\begin{tabular}{|c|c|c|c|c|}
\hline Compound & $\begin{array}{l}\beta \text {-carotene/ linoleic acid assay } \\
\mathrm{IC}_{50}(\mu \mathrm{M})\end{array}$ & $\begin{array}{l}\text { DPPH' assay } \\
\mathrm{IC}_{50}(\mu \mathrm{M})\end{array}$ & $\begin{array}{l}\text { ABTS }^{++} \text {assay } \\
\mathrm{IC}_{50}(\mu \mathrm{M})\end{array}$ & $\begin{array}{l}\text { CUPRAC } \\
\mathrm{A}_{0.5}(\mu \mathrm{M})\end{array}$ \\
\hline 1 & $52.96 \pm 0.74$ & $49.31 \pm 0.48$ & $56.03 \pm 0.31$ & $37.68 \pm 0.02$ \\
\hline 2 & $40.39 \pm 0.80$ & $41.43 \pm 0.77$ & $43.19 \pm 0.84$ & $29.94 \pm 0.01$ \\
\hline 3 & $21.80 \pm 0.69$ & $18.21 \pm 0.75$ & $19.20 \pm 0.25$ & $10.11 \pm 0.00$ \\
\hline 4 & $44.37 \pm 0.17$ & $43.94 \pm 0.11$ & $45.92 \pm 0.87$ & $31.32 \pm 0.01$ \\
\hline 5 & $39.02 \pm 0.66$ & $36.17 \pm 0.89$ & $39.21 \pm 0.34$ & $26.39 \pm 0.02$ \\
\hline 6 & $32.58 \pm 0.31$ & $28.94 \pm 0.52$ & $32.27 \pm 0.06$ & $21.77 \pm 0.00$ \\
\hline 7 & $35.07 \pm 0.55$ & $32.19 \pm 0.08$ & $35.80 \pm 0.72$ & $24.28 \pm 0.02$ \\
\hline 8 & $30.14 \pm 0.33$ & $25.81 \pm 0.33$ & $30.03 \pm 0.76$ & $19.22 \pm 0.00$ \\
\hline 9 & $29.07 \pm 0.52$ & $24.09 \pm 0.36$ & $27.06 \pm 0.49$ & $15.09 \pm 0.03$ \\
\hline 10 & $26.49 \pm 0.61$ & $22.16 \pm 0.51$ & $23.20 \pm 0.37$ & $13.47 \pm 0.01$ \\
\hline 11 & $47.34 \pm 0.19$ & $47.16 \pm 0.20$ & $51.37 \pm 0.44$ & $35.64 \pm 0.00$ \\
\hline 12 & $43.09 \pm 0.73$ & $46.44 \pm 0.34$ & $49.54 \pm 0.63$ & $34.03 \pm 0.01$ \\
\hline 13 & $41.86 \pm 0.52$ & $38.29 \pm 0.99$ & $40.17 \pm 0.65$ & $34.61 \pm 0.00$ \\
\hline 14 & $32.67 \pm 0.11$ & $29.34 \pm 0.95$ & $32.38 \pm 0.15$ & $27.82 \pm 0.00$ \\
\hline 15 & $16.28 \pm 0.30$ & $8.39 \pm 0.87$ & $9.38 \pm 0.93$ & $5.75 \pm 0.01$ \\
\hline 16 & $34.07 \pm 0.89$ & $33.29 \pm 0.23$ & $34.92 \pm 0.16$ & $29.13 \pm 0.00$ \\
\hline 17 & $30.12 \pm 0.27$ & $25.65 \pm 0.18$ & $30.71 \pm 0.55$ & $22.29 \pm 0.01$ \\
\hline 18 & $25.87 \pm 0.30$ & $19.31 \pm 0.66$ & $23.96 \pm 0.24$ & $16.50 \pm 0.03$ \\
\hline 19 & $26.32 \pm 0.18$ & $22.92 \pm 0.44$ & $26.74 \pm 0.66$ & $19.36 \pm 0.00$ \\
\hline 20 & $22.39 \pm 0.79$ & $18.49 \pm 0.37$ & $21.35 \pm 0.14$ & $14.79 \pm 0.01$ \\
\hline 21 & $21.26 \pm 0.08$ & $16.70 \pm 0.51$ & $19.88 \pm 0.75$ & $11.13 \pm 0.00$ \\
\hline 22 & $19.17 \pm 0.34$ & $13.39 \pm 0.07$ & $14.79 \pm 0.26$ & $9.47 \pm 0.00$ \\
\hline
\end{tabular}

${ }^{a}$ Values expressed are means \pm S.E.M. of three parallel measurements. $p<0.05$, significantly different with student's $t$-test.

${ }^{\mathrm{b}}$ Reference compound 


\begin{tabular}{|c|c|c|c|c|}
\hline Compound & $\begin{array}{l}\beta \text {-carotene/ linoleic acid assay } \\
\mathrm{IC}_{50}(\mu \mathrm{M})\end{array}$ & $\begin{array}{l}\text { DPPH assay } \\
\mathrm{IC}_{50}(\mu \mathrm{M})\end{array}$ & $\begin{array}{l}\text { ABTS }^{++} \text {assay } \\
\mathrm{IC}_{50}(\mu \mathrm{M})\end{array}$ & $\begin{array}{l}\text { CUPRAC } \\
\mathrm{A}_{0.5}(\mu \mathrm{M})\end{array}$ \\
\hline 23 & $39.78 \pm 0.51$ & $37.62 \pm 0.43$ & $36.21 \pm 0.61$ & $33.07 \pm 0.02$ \\
\hline 24 & $37.55 \pm 0.60$ & $36.81 \pm 0.32$ & $35.99 \pm 0.30$ & $31.37 \pm 0.03$ \\
\hline $\mathrm{BHT}^{\mathrm{b}}$ & $2.34 \pm 0.09$ & $54.97 \pm 0.99$ & $2.91 \pm 0.55$ & $3.80 \pm 0.02$ \\
\hline$a-T_{O C}^{b}$ & $4.50 \pm 0.09$ & $12.26 \pm 0.07$ & $4.87 \pm 0.45$ & $40.48 \pm 0.02$ \\
\hline \multicolumn{5}{|c|}{$\begin{array}{l}\text { a Values expressed are means } \pm \text { S.E.M. of three parallel measurements. } p<0.05 \text {, significantly different } \\
\text { with student's } t \text {-test. }\end{array}$} \\
\hline \multicolumn{5}{|c|}{${ }^{b}$ Reference compound } \\
\hline
\end{tabular}

\section{Figures}

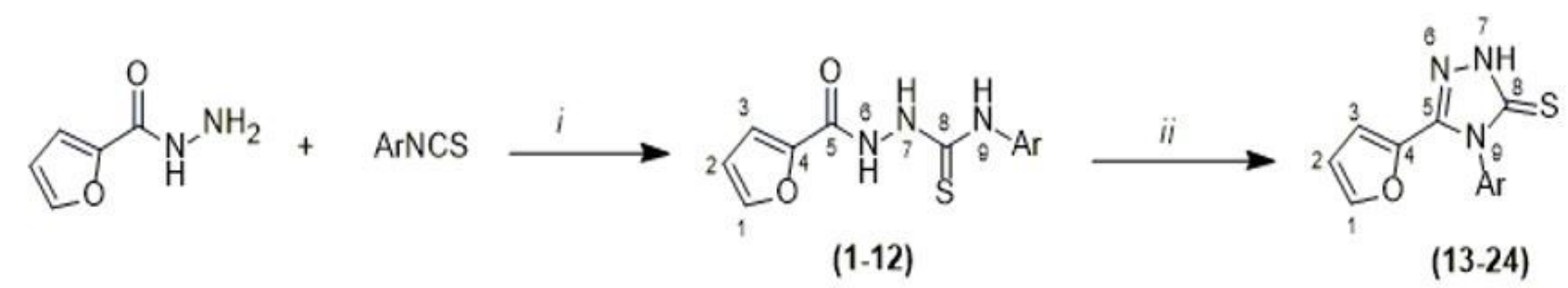

$\mathrm{Ar}$

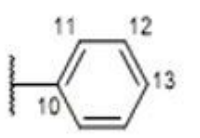

(1 and 13)

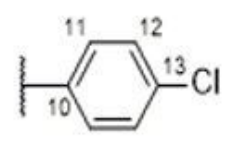

(2 and 14)

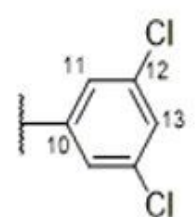

(3 and 15)

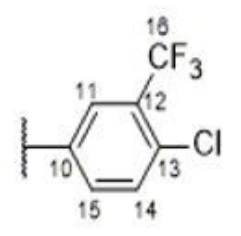

(4 and 16)

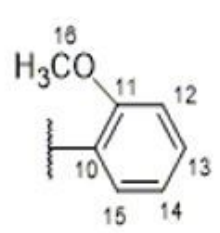

(5 and 17)

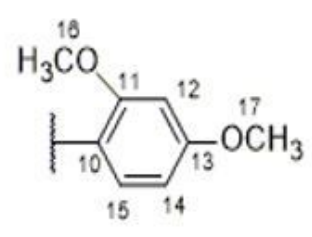

(6 and 18)

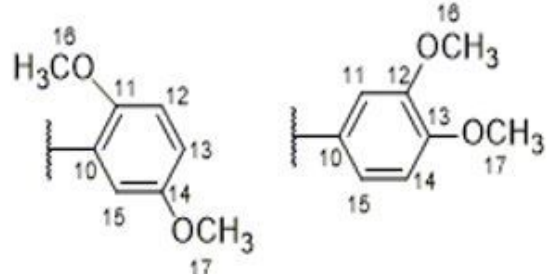

(7 and 19)
(8 and 20)<smiles>COc1cc(I)cc(I)c1</smiles>

(9 and 21)

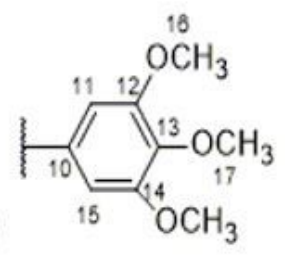

(10 and 22)<smiles>CN(C)c1ccc(I)cc1</smiles>

(11 and 23)<smiles>CCN(CC)c1ccc(I)cc1</smiles>

(12 and 24)

Figure 1 
Synthetic pathway of thiosemicarbazides (1-12) and 1,2,4-triazoles (13-24). Reagents and conditions: (a) DCM, rt.; (b) EtOH, reflux.

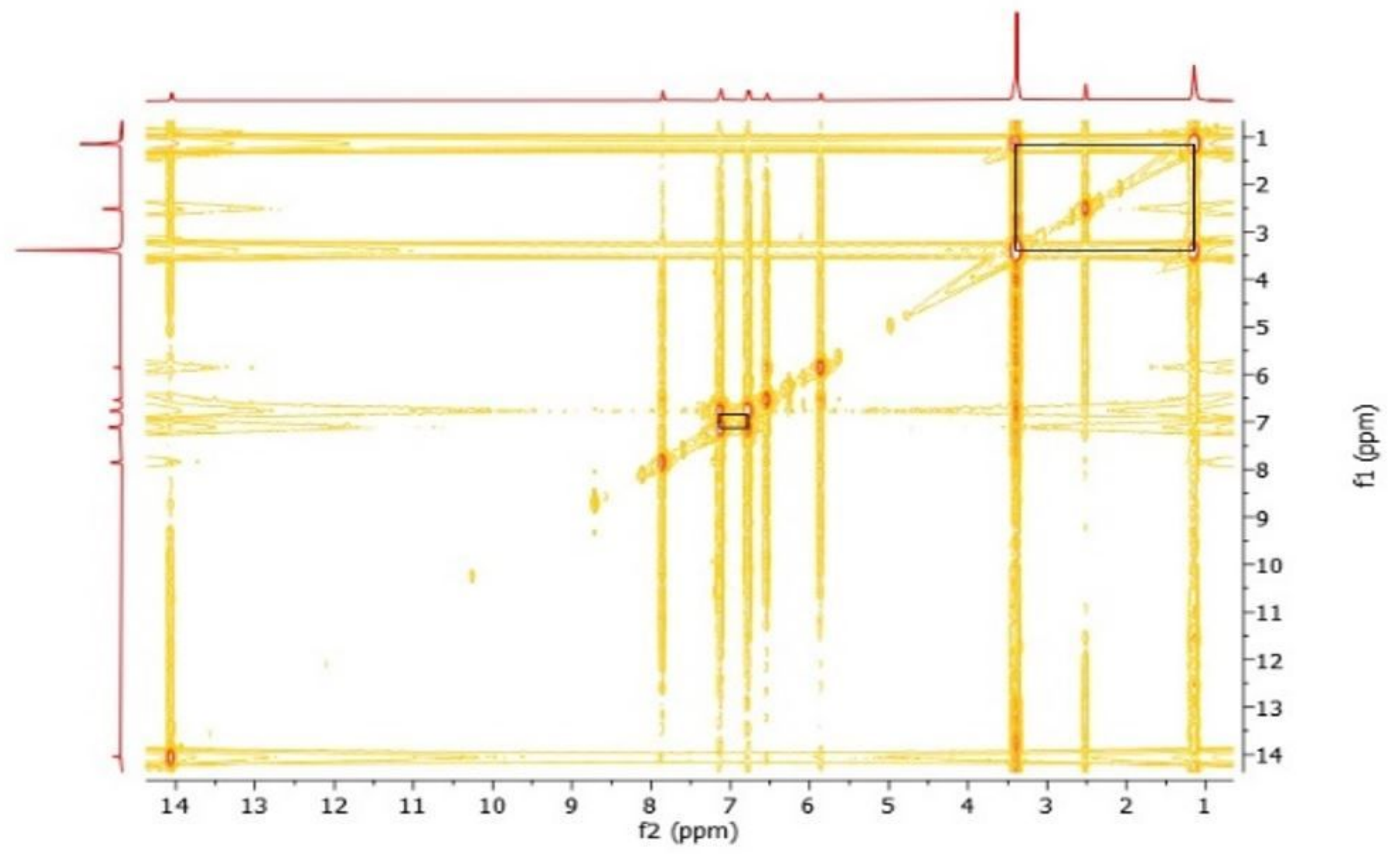

Figure 2

The COSY spectrum of compounds 24 


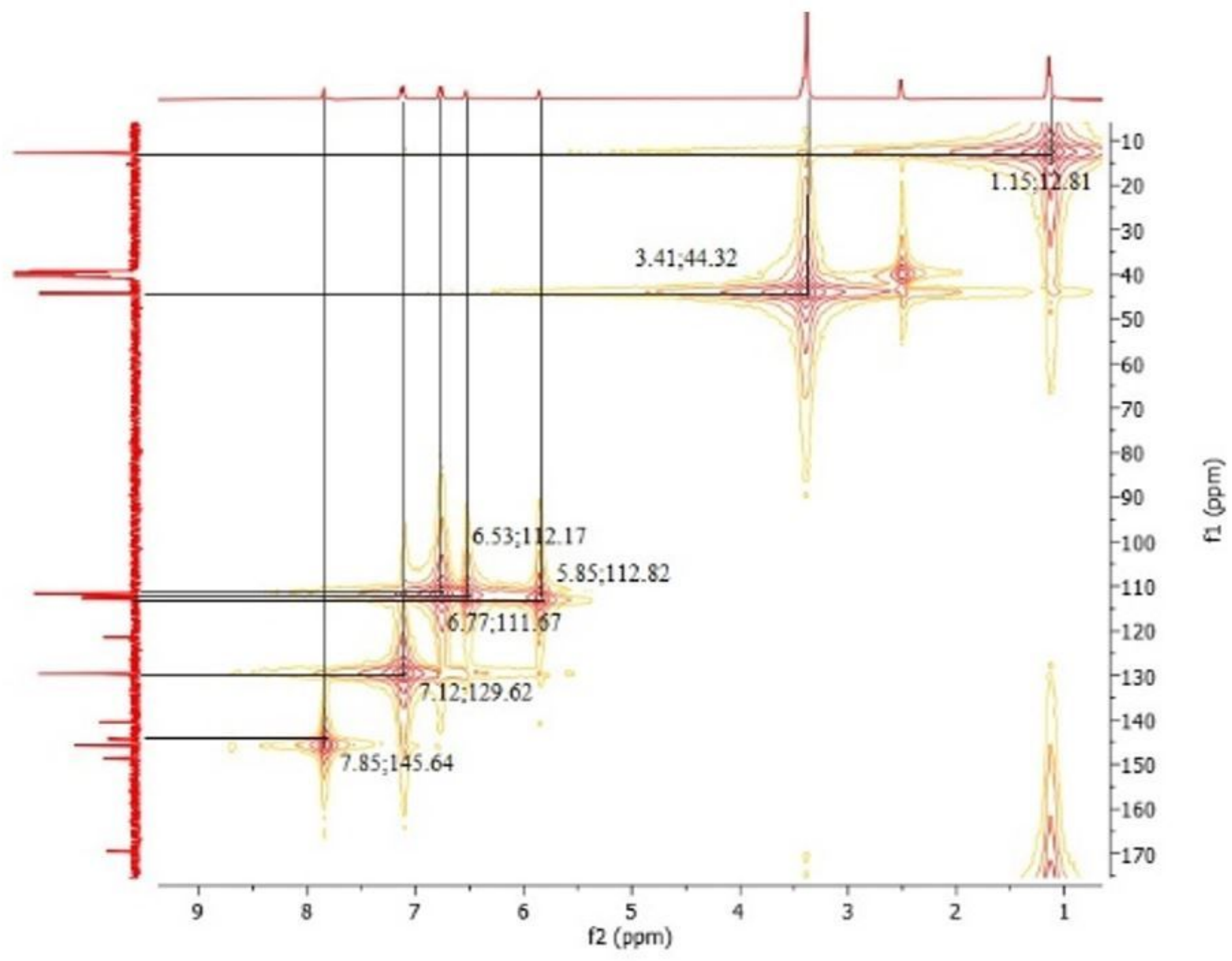

Figure 3

COSY and HETCOR spectrum of compound 13 


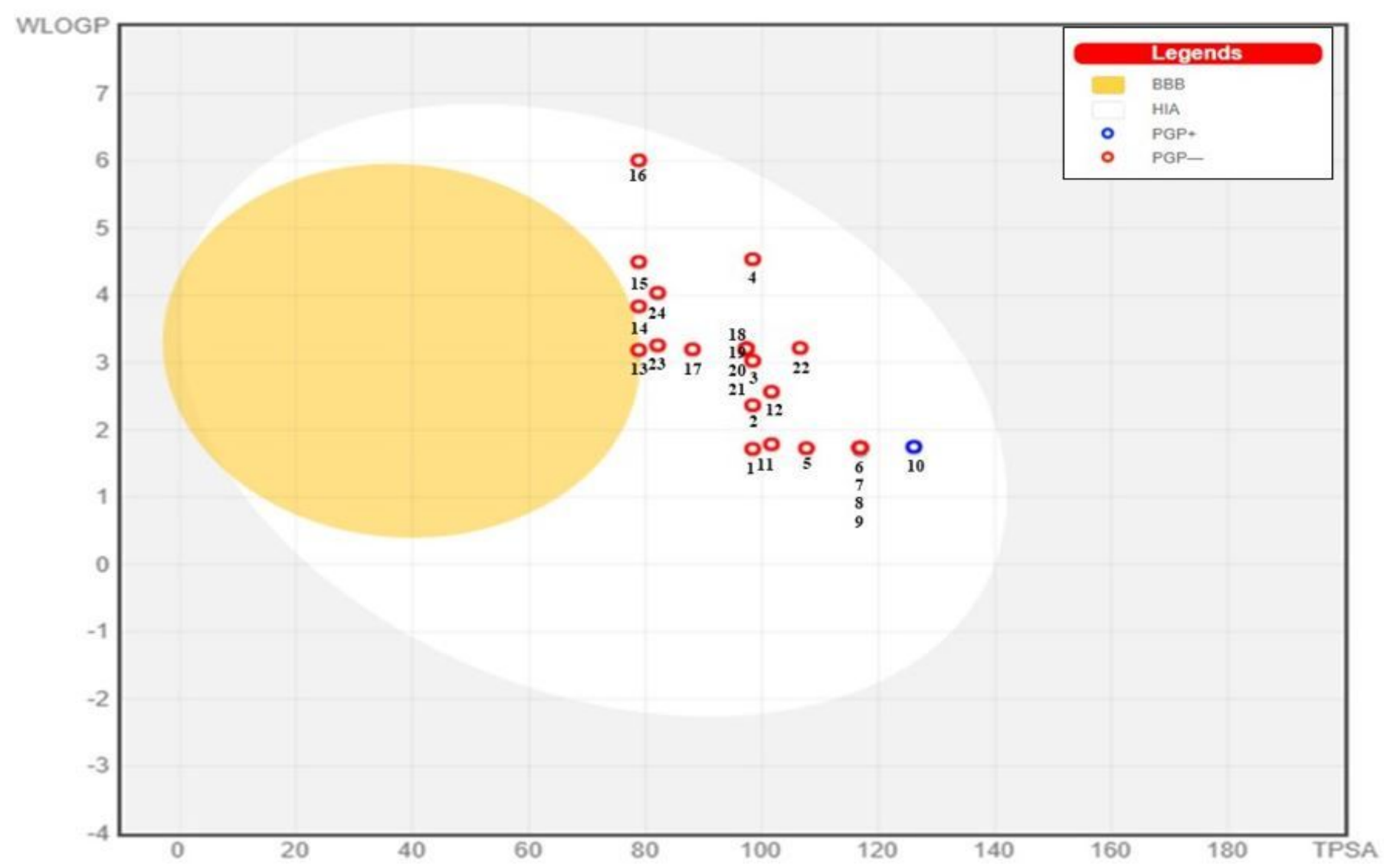

\section{Figure 4}

Graphical distribution of synthesized thiosemicarbazides 1-12 and 1,2,4-triazoles 13-24 and enzyme inhibitor standards according to the BOILED-EGG predictive model Main text paragraph.

\section{Supplementary Files}

This is a list of supplementary files associated with this preprint. Click to download.

- GraphicalAbstract.jpg

- Supplementaryitem.docx 